\title{
Depletion in post-AGB stars with a dusty disc. II. ${ }^{\star \star \star}$
}

\author{
T. Maas ${ }^{1,2}$, H. Van Winckel ${ }^{2}$, and T. Lloyd Evans ${ }^{3}$ \\ 1 Department of Astronomy, University of Texas, Austin, TX 78712, USA \\ e-mail: thomas@astro.as.utexas.edu \\ 2 Instituut voor Sterrenkunde, KU Leuven, Celestijnenlaan 200B, 3001 Leuven, Belgium \\ ${ }^{3}$ School of Physics and Astronomy, University of St Andrews, North Haugh, St Andrews, Fife, Scotland KY16 9SS, UK
}

Received 19 July 2004 / Accepted 30 August 2004

\begin{abstract}
We present a chemical abundance analysis on the basis of high signal-to-noise and high resolution spectra for 12 stars of our newly defined sample. The selection criterion for the stars of this sample was their position in the "RV Tauri" box in the [12] - [25], [25] - [60] two-colour diagram. An additional requisite was the presence of a near-IR excess. We found that the photospheres of nine stars are affected by the depletion process. Since the most likely origin of the near-IR excess is a disc, this strongly suggests that the phenomenon of depletion and the presence of a disc are linked. Among the depleted stars we find different depletion patterns. In seven stars elements with a condensation temperature below $1500 \mathrm{~K}$ are not or only slightly affected. Large underabundances are measured only for most elements with a condensation temperature above $1500 \mathrm{~K}$.
\end{abstract}

Key words. stars: abundances - stars: AGB and post-AGB - stars: evolution - stars: binaries: spectroscopic

\section{Introduction}

RV Tauri stars form a loosely defined class of variable stars with a characteristic light curve showing alternating deep and shallow minima. They have "formal" periods (the time between two successive deep minima) between 30 and 150 days and occupy the high luminosity end of the Pop II Cepheids. Gehrz (1972) and Lloyd Evans (1985) pointed out that many RV Tauri stars show near-IR excesses. Jura (1986) classified RV Tauri stars as post-AGB stars since many objects were shown to be surrounded by considerable amounts of circumstellar dust as detected by IRAS. Lloyd Evans (1985) and Raveendran (1989) showed that the RV Tauri stars are located in a well-defined and relatively thinly-populated part of the IRAS colour-colour diagram. The defining rectangle is

$\left\{\begin{array}{l}{[12]-[25] \equiv 1.56+2.5 \log [F(25) / F(12)]=1.0-1.55} \\ {[25]-[60] \equiv 1.88+2.5 \log [F(60) / F(25)]=0.20-1.0 .}\end{array}\right.$

This is the second in a series of papers on a new sample of postAGB stars. They were selected by Lloyd Evans (1999, and in preparation) on the basis of their IRAS colours, which place them in the "RV Tauri" box in the [12] - [25], [25] - [60] twocolour diagram. A few known RV Tauri stars fall in an extended region to the red, which was less intensively searched for new examples. IRAS $17038-4815$ and IRAS $20056+1834$ fall in this

\footnotetext{
* Based on observations collected at the European Southern Observatory in Chile (64.L-117 and 67.D-0054) and at the Swiss Euler telescope at La Silla, Chile.

$\star \star$ Tables 2, 4-7, 9, Figs. 3-5, 7 and Appendix are only available in electronic form at http://www. edpsciences.org
}

extension (Lloyd Evans, in preparation). An additional selection criterion is an observed excess in the L-band, indicative of a dusty disc (Lloyd Evans 1997). Both criteria lead to a typical spectral energy distribution with a broad IR excess (see Fig. 1). In previous years we have been accumulating different data sets, each probing different physical properties of the stars. Initial photometric monitoring in the optical and near-IR at SAAO were supplemented with radial velocity monitoring at La Silla. Beside that, we obtained high quality spectra with FEROS and CO emission spectra with SEST, both at La Silla. The purpose is to characterize these evolved objects and to compare them with GCVS RV Tauri stars. We want to investigate the possible relation between the specific SED characteristics; the binary nature of the central object and the presence of chemical peculiarities. The latter is a widespread phenomenon among GCVS RV Tauri stars. Many objects display depletion patterns and mimic in their photospheric pattern, the abundances of the gas phase of the Interstellar Medium (ISM): species with a high dust condensation temperature are underabundant relative to non-refractory elements which have a low dust condensation temperature.

In our first paper on this sample (Maas et al. 2003) we highlighted one object, IRAS 08544-4431. Its spectral energy distribution shows a broad IR excess starting already at $H$. The spectral type of F3 places it well to the high-temperature side of the instability strip (Lloyd Evans 1999, and in preparation), and the photometric peak-to-peak amplitude of $\Delta V=0.17 \mathrm{mag}$ is much smaller than those of recognised RV Tauri stars. Our radial velocity monitoring revealed it to be a binary with an orbital period of $503 \pm 2$ days. Furthermore, IRAS 08544-4431 
Table 1. Basic data for the programme stars. Besides the IRAS name, the spectral type (determined on the basis of low resolution spectra (Lloyd Evans, in preparation)), the Equatorial coordinates, $\left(\alpha_{2000}, \delta_{2000}\right)$, the galactic coordinates, $(l, b)$, the visual magnitude, $V$ (Maas et al., in preparation), and the 2MASS $K$-magnitude are given. Notes: $\mathrm{p}$ for peculiar spectral features, $(\mathrm{R})$ for the presence of enhanced $\mathrm{CH}$ and CN bands, De for strong emission in the Na D line (Lloyd Evans 1999, and in preparation) and ":" for poor photometry.

\begin{tabular}{l|lcccrrr}
\hline \hline IRAS & sp. type & \multicolumn{1}{c}{$\alpha_{2000}$} & \multicolumn{1}{c}{$\delta_{2000}$} & $l$ & \multicolumn{1}{c}{$b$} & \multicolumn{1}{c}{$V$} \\
\hline $08544-4431$ & F3 & 085614.18 & -444310.7 & 265.51 & +0.39 & 9.1 & $3.52:$ \\
$09060-2807$ & F5 & 090810.14 & -281910.4 & 254.59 & +12.94 & 11.3 & 6.95 \\
$09144-4933$ & G0 & 091609.64 & -494612.1 & 271.51 & -0.50 & $11.1:$ & 6.37 \\
$09538-7622$ & G0 & 095357.67 & -763652.3 & 293.17 & -17.24 & 11.7 & 7.72 \\
$15469-5311$ & F3 & 155043.80 & -532043.3 & 327.82 & +0.63 & 10.6 & 4.97 \\
$16230-3410$ & F8 & 162620.39 & -341712.9 & 345.54 & +10.26 & 11.4 & 7.81 \\
$17038-4815$ & G2p(R) & 170736.66 & -481908.5 & 339.79 & -4.67 & 10.6 & 6.52 \\
$17233-4330$ & G0p(R) & 172658.64 & -433313.6 & 345.64 & -4.69 & 12.1 & 8.37 \\
$17243-4348$ & G2 & 172753.63 & -435046.3 & 345.50 & -5.00 & 10.4 & 6.46 \\
$19125+0343$ & F2 & 191501.17 & +034842.7 & 39.02 & -3.49 & 10.2 & $5.65:$ \\
$19157-0247$ & F3 & 191822.71 & -024210.8 & 33.59 & -7.22 & 10.7 & 7.02 \\
$20056+1834$ & G0 De & 200754.60 & +184254.8 & 58.44 & -7.46 & 12.5 & 7.47 \\
\hline
\end{tabular}

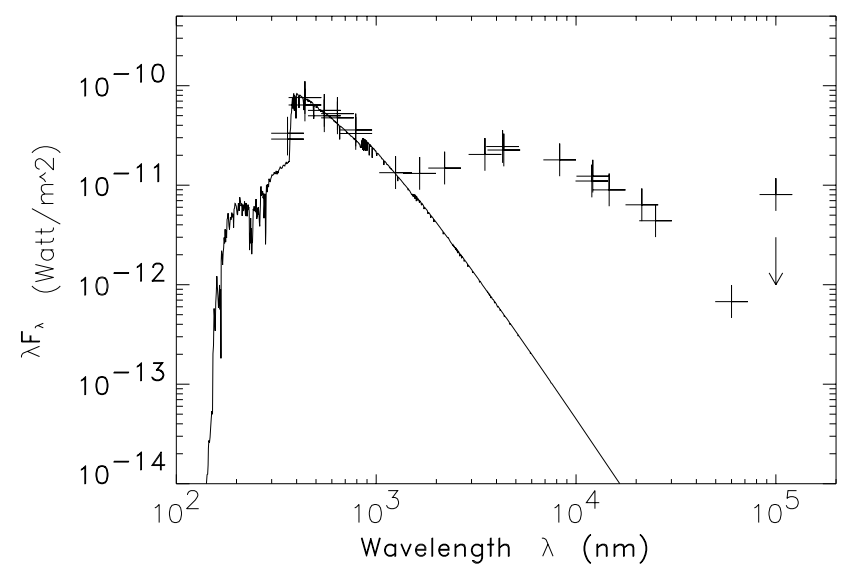

Fig. 1. The spectral energy distribution (SED) of IRAS 15469-5311. The SED is typical for the sample, showing a large and broad IR-excess. A total reddening of $E(B-V)=1.5$ was deduced minimizing the difference between the deredenned optical fluxes (plus signs) and the model atmosphere (full line).

is detected in both the CO (2-1) and (1-0) mm-wave emission lines. The triangular shape of the weak $\mathrm{CO}$ profile confirms that part of the circumstellar material is not freely expanding but resides probably in a dusty circumbinary disc. Moreover, our chemical abundance analysis of a high resolution spectrum of high $S / N$ revealed that a depletion process has modified the photospheric abundances to a moderate extent $([\mathrm{Zn} / \mathrm{Fe}]=+0.4)$. All these findings confirm that the F-type star IRAS 08544-4431 is a binary post-AGB star surrounded by a dusty disc.

In this paper we report on our chemical abundance analysis of the high resolution and high signal-to-noise spectra of the stars of our sample with spectral type F and G. They are listed in Table 1. The positions of the optical sources agree well with the positions of the 2MASS sources (Lloyd Evans, in preparation). IRAS 16230-3410, IRAS 17038-4815, IRAS 172334330 and IRAS 17243-4348 were classified by Lloyd Evans (1999, and in preparation) as genuine RV Tauri stars. Low resolution spectra of IRAS 17038-4815 and IRAS 17233-4330 show strong $\mathrm{CN}$ and $\mathrm{CH}$ bands with respect to the rest of the spectrum. Both stars belong to the spectroscopic RV Tauri group RVB (for the definition of the different spectroscopic classes of RV Tauri stars, see Preston et al. 1963; Maas et al. 2002). The low resolution spectra of IRAS 16230-3410 and IRAS 17243-4348 are typical for members of the spectroscopic group RVA (Lloyd Evans, 1999 and in preparation). Since IRAS 09538-7622 has a high peak-to-peak photometric amplitude and a spectral type $\mathrm{G}$, we also consider it to be a genuine RV Tauri (Maas et al., in preparation). It belongs to the spectroscopic group RVA (Lloyd Evans 1999, and in preparation).

Almost all objects listed in Table 1 are virtually unstudied prior to our research efforts. Only for IRAS 17243-4348 and IRAS 20056+1834 is there a substantial literature. IRAS 17243-4348, also known as LR Sco, was misclassified as an R Coronae Borealis star by Stephenson (1978), and was classified to be a yellow supergiant by Giridhar et al. (1990). In this paper, we classify the object as a low mass evolved object. Menzies \& Whitelock (1988) observed IRAS 20056+1834 (QY Sge) and discovered the near infrared excess and the $\mathrm{Na}$ D emission superimposed on a spectrum of G0 I; they adopted a model in which the star is seen by reflection from circumstellar matter while direct light is obscured by dust, as suggested by Herbig (1970). Rao et al. (2002) proposed a disk torus geometry for the circumstellar dust, with a bipolar flow which is the site for formation of emission lines from low levels of abundant neutral metal atoms. The abundance analysis of high resolution spectra by Rao et al. (2002) revealed that the chemical composition is approximately solar except for highly condensable elements which are depleted by factors of 5-10, on the basis of which it is suggested that QY Sge is a genuine RV Tauri star. In addition, its temperature and luminosity fall in the same range as do those of the RV Tauri stars.

The outline of this paper is as follows. First, we give an overview of the observations, after which we make a short interlude on the $\mathrm{H} \alpha$-profile in the spectra. In Sect. 4 we specify how we determined the photospheric model parameters and discuss the errors on the abundances due to the uncertainties on the model parameters. In Sect. 5 we present the abundances. 
We look for traces of the depletion process and/or nucleosynthesis processes in the deep layers of the stars, followed by dredge-up. In Sect. 6 we discuss the observed abundance patterns and compare them with those observed in other depleted stars. We end with the conclusions. A detailed modeling of the spectral energy distribution of these stars will be discussed in a forthcoming paper (De Ruyter et al., in preparation).

\section{Observations}

The spectra were almost all obtained by H. Van Winckel with the Fiber-Fed Extended Range Optical Spectrograph (FEROS) mounted on the $1.52 \mathrm{~m}$ telescope at ESO, La Silla (Chile) in the ESO periods \#64 (22-24 March 2000) and \#67 (26-28 March 2001). A spectrum of IRAS 08544-4431 was already previously obtained with FEROS by G. Meeus during the ESO Period \#62. This spectrograph covers the complete optical wavelength domain (3700-8600 $⿱$ ) and has a resolution of 48000 . Two fibers feed simultaneously the spectrograph. While the first fiber transmits the light of the object, the second is fed by the background sky. The spectrograph is equipped with a CCD camera incorporating a monolithic thinned and backside-illuminated CCD chip by EEV with $2048 \times 4096$ pixels of $15 \times 15 \mu \mathrm{m}$ size, which is cooled to a temperature between -150 and $-80{ }^{\circ} \mathrm{C}$. For this analysis we used the pipeline reduced spectra. The pipeline reduction consists of background subtraction, flat fielding, order extraction and wavelength calibration. The normalisation of the spectra was done by fitting a smoothed spline through interactively chosen continuum points. For IRAS 09538-7622 we do not have a FEROS spectrum at our disposal. Since we are monitoring this star with the spectrograph CORALIE mounted on the Swiss Euler telescope at La Silla, we analysed the CORALIE spectrum with the highest $S / N$. An overview of the obtained spectra is presented in Table 2. For IRAS 20056+1834 we do not have a good FEROS spectrum either. We will use the results of Rao et al. (2002) throughout the paper.

\section{H $\alpha$-profile}

The $\mathrm{H} \alpha$-line at $6562.8 \AA$ of the programme stars shows a wide variety of profiles (see Fig. 2). All profiles, except that of IRAS 17233-4330 show an emission component (the double absorption profile of IRAS 17243-4348 is very likely due to a central emission superimposed on a wider absorption profile). In the stars IRAS 08544-4431, IRAS 15469-5311, (IRAS 16230-3410), IRAS 17038-4815， IRAS 19125+0343 and IRAS 19157-0247, a P-Cygni profile is seen, giving evidence for ongoing mass loss. The blue shifted absorption in these profiles points to a very high velocity outflow. The massloss mechanism in these stars is, however, not well understood: the stars have spectral type $\mathrm{F}$ and $\mathrm{G}$ and are expected to be too cool to develop significant mass loss by line-driven winds. Nevertheless, this mass loss in the early post-AGB phase could play an important role in the shaping of post-AGB nebulae and planetary nebulae. We refer to a detailed analysis of the profile variability of IRAS 08544-4431 in Maas et al. (2003). A

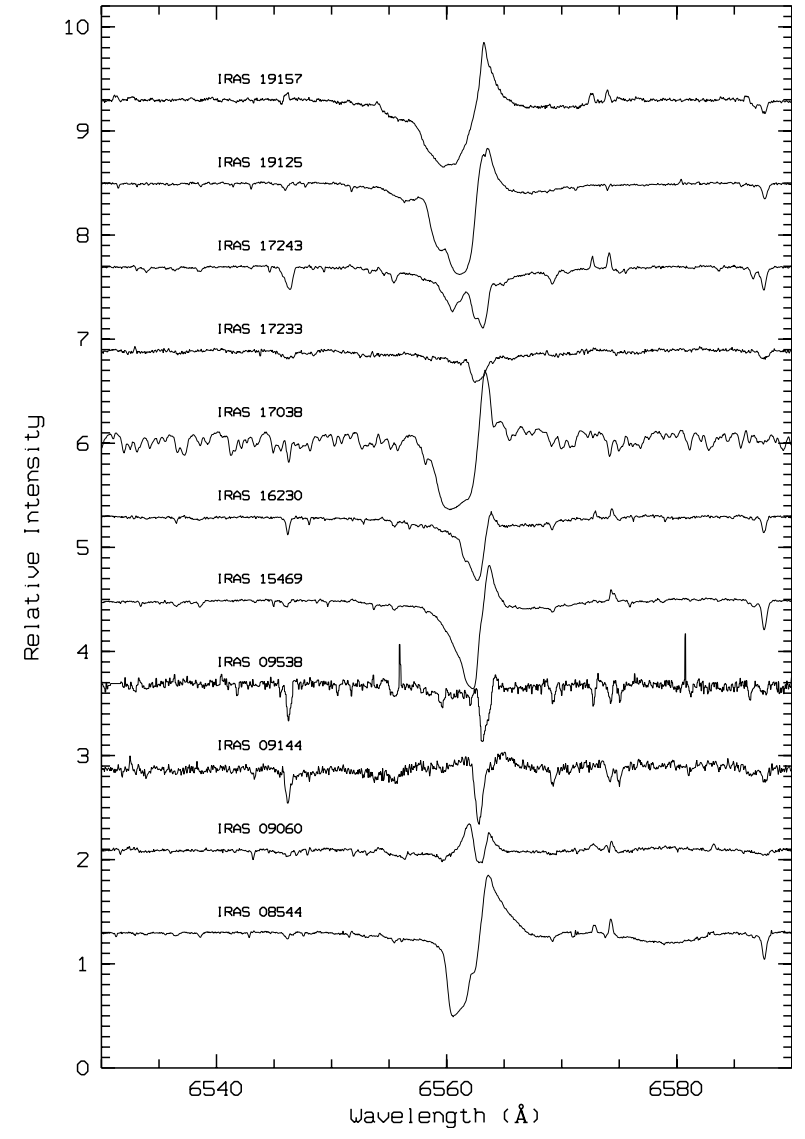

Fig. 2. The $\mathrm{H} \alpha$-profile for all programme stars.

more quantitative analysis of the $\mathrm{H} \alpha$ profile and stellar wind is beyond the scope of this paper but will certainly be rewarding.

\section{Analysis}

\subsection{Determination of the model parameters and abundances}

For the abundance analysis we used the models of Kurucz (1993). The abundances are calculated with the LTE chemical abundance programme MOOG of C. Sneden (version April 2002).

To derive precise abundances, accurate oscillator strengths (log $g f$-values) are of utmost importance. Over many years of research, a considerable list of lines with good oscillator strengths has been accumulated at the Instituut voor Sterrenkunde, KULeuven. For more details see Van Winckel \& Reyniers (2000).

The model parameters: the effective temperature, $T_{\text {eff }}$, the (logarithm of) surface gravity, $\log g$, and the microturbulent velocity, $\xi_{\mathrm{t}}$, are determined on the basis of the spectral lines of Fe.

The effective temperature is determined by demanding that the iron abundance, derived from the Fe I-lines, is independent of the excitation potential. This method becomes less accurate due to non-LTE effects for the hotter stars in our sample $\left(T_{\text {eff }} \geq 7500 \mathrm{~K}\right)$ : IRAS 15469-5311, IRAS 19125+0343 and IRAS 19157-0247. For these stars we used the Fe II lines to determine $T_{\text {eff. }}$ For IRAS 19157-0247 this was even the only 
Table 3. The model parameters for the programme objects. For IRAS 15469-5311 we used the listed model parameters for both spectra. The model parameters for IRAS $20056+1834$ are taken from Rao et al. (2002).

\begin{tabular}{c|ccrc}
\hline \hline IRAS & $T_{\text {eff }}(\mathrm{K})$ & $\log g(\mathrm{cgs})$ & {$[\mathrm{Fe} / \mathrm{H}]$} & $\xi_{\mathrm{t}}\left(\mathrm{km} \mathrm{s}^{-1}\right)$ \\
\hline $08544-4431$ & 7250 & 1.5 & -0.5 & 4.5 \\
$09060-2807$ & 6500 & 1.5 & -0.5 & 5.0 \\
$09144-4933$ & 5750 & 0.5 & -0.5 & 4.0 \\
$09538-7622$ & 5500 & 1.0 & -0.5 & 5.5 \\
$15469-5311$ & 7500 & 1.5 & 0.0 & 5.0 \\
$16230-3410$ & 6250 & 1.0 & -0.5 & 4.0 \\
$17038-4815$ & 4750 & 0.5 & -1.5 & 5.0 \\
$17233-4330$ & 6250 & 1.5 & -1.0 & 5.0 \\
$17243-4348$ & 6250 & 0.5 & 0.0 & 5.0 \\
& 5250 & 0.0 & 0.0 & 5.0 \\
$19125+0343$ & 7750 & 1.0 & -0.5 & 4.0 \\
$19157-0247$ & 7750 & 1.0 & 0.0 & 3.0 \\
$20056+1834$ & 5850 & 0.7 & -0.4 & 4.5 \\
\hline
\end{tabular}

possibility as the low excitation Fe I lines are all in emission making the excitation range of Fe I absorption lines too small to derive a temperature. As an upper limit indicator for the temperature at a given gravity, the presence of He-lines in the spectrum can be used. These lines appear in the spectrum above a temperature of $7750 \mathrm{~K}$ (depending on the gravity). Only in IRAS $19125+0343$ the He-line at $5875.6 \AA$ is marginally detected.

The surface gravity is fixed by postulating an equal abundance derived from Fe I and Fe II lines and the microturbulent velocity by demanding the independence of the abundance, derived from the $\mathrm{Fe} I$ lines, on the reduced equivalent width $(E W / \lambda)$.

The derived parameters are shown in Table 3. The derived temperature and gravities confirm the results of Lloyd Evans (1999) that the programme stars have a high luminosity and that their temperatures put them in the instability strip or just at the blue side of it. Moreover, the low surface gravities of the programme stars are in agreement with the long pulsation time scales of most of them (Maas et al., in preparation).

Typical errors on the derived model parameters are: $\Delta T_{\text {eff }}=$ $250 \mathrm{~K}, \Delta \log g=0.5, \Delta \xi_{\mathrm{t}}=1.0 \mathrm{~km} \mathrm{~s}^{-1}$. We study the influence of these parameter uncertainties on the abundances in the next section.

For each ion we looked for unblended lines with an $E W<$ $150 \mathrm{~m} \AA$. We used the VALD2 database (Kupka et al. 1999) to check for blends. When the abundance of a line exceeded the average abundance of the other lines of the same atom by 0.2 dex, we calculated the expected central depths of all lines in the neighbourhood, adopting the abundances determined by other unblended lines and the model parameters of the star. Next, we determined the $E W$ s of the lines of which the central wavelength differed less than $0.3 \AA$ with that of the considered line, adopting the abundances determined by other lines. In many cases, unresolved blends were found in this way.
Because of the presence of strong interstellar bands in the spectra of most programme stars (see Fig. 3), we also examined the possible contamination of the EWs of the absorption lines, which were used in the analysis, by these bands.

We give a small general overview of the typical problems and characteristics of the abundance determination of a given chemical element.

The carbon $(\mathrm{C})$-abundance is determined for all stars. This abundance is mainly based on the high excitation multiplet at $7100 \AA$ (except for IRAS 09060-2807, IRAS 09538-7622 and IRAS 17038-4815, because of the low C-abundance, limited available wavelength domain and low temperature, respectively).

Good nitrogen(N)-lines are sparse in the spectra. For only five objects a $\mathrm{N}$-abundance was determined, based on an average of 2 lines. Also for oxygen $(\mathrm{O})$ not many lines are present. The oxygen-triplet with excitation temperature of $10.74 \mathrm{eV}$ around $6156 \AA$ shows up only in the hotter stars of the sample (see Fig. 4). The forbidden oxygen lines at $6300 \AA$ and $6364 \AA$ are in emission in all programme stars. The NLTE sensitive red triplet was never used.

Almost always two low excitation neutral lines are used for the sodium(Na)-abundance.

The sulfur(S)-abundance is based on some or all lines of the two triplets around $4695 \AA$ and $6750 \AA$ (see Fig. 5). The S-lines in the wavelength range 8660-8700 $\AA$ turned out to be inappropriate because of the very poor quality of our spectra in this wavelength domain.

One or two lines are used for the zinc ( $\mathrm{Zn}$ ) abundance. In total 4 different $\mathrm{Zn}$-lines are used, of which the lines at $4722 \AA$ and $4810 \AA$ are most often available.

Elements with neutral and singly ionised lines offer an extra check for the reliability of the model parameters. For magnesium $(\mathrm{Mg})$, silicon $(\mathrm{Si})$, calcium $(\mathrm{Ca})$, titanium $(\mathrm{Ti})$, manganese (Mn) and nickel (Ni) sometimes neutral and singly ionised lines are available, but mostly there is only one line present in one of the two ionisation stages. However, for chromium $(\mathrm{Cr})$ a considerable number of lines in both ionisation stages is often present.

For all stars a scandium(Sc)-abundance is determined.

For copper $(\mathrm{Cu})$, aluminium $(\mathrm{Al})$ and the r-process element (Eu), an abundance is sometimes derived.

There are few s-process lines detected. These atoms are expected to be mainly singly ionised in the $T_{\text {eff }}$ range of our programme stars. Often only an upper limit for the abundance could be determined. This upper limit is derived from the strongest predicted line, which is not present in the spectrum, ascribing it an $E W$ of $5 \mathrm{~m} \AA$.

\subsection{Abundance uncertainties}

The line-to-line scatter, which is the standard deviation of the mean, is given for the abundance derived from each ion and reflects the uncertainties which result from the errors of the determined equivalent widths, the $\log (g f)$-values and the continuum placement. The lines of most ions have excitation temperatures in a small range. Therefore the influence of the uncertainties 
Table 8. The synopsis of the abundance results. $[\mathrm{Fe} / \mathrm{H}]_{0}$ is the estimated initial metallicity on the basis of the $\mathrm{Zn}$-abundance, if this abundance was believed to be unaffected. For the initial metallicity determination we made use of the relation: $[\mathrm{Zn} / \mathrm{Fe}]=+0.04$ from Sneden et al. $(1991)$.

\begin{tabular}{rrrrrrrrrrrr}
\hline \hline IRAS & {$[\mathrm{Fe} / \mathrm{H}]$} & {$[\mathrm{Fe} / \mathrm{H}]_{0}$} & {$[\mathrm{C} / \mathrm{Fe}]$} & {$[\mathrm{N} / \mathrm{Fe}]$} & {$[\mathrm{O} / \mathrm{Fe}]$} & $\mathrm{C} / \mathrm{O}$ & {$[\mathrm{Zn} / \mathrm{Fe}]$} & {$[\mathrm{S} / \alpha]$} & {$[\mathrm{S} / \mathrm{Ti}]$} & {$[\mathrm{Zn} / \mathrm{Ti}]$} & {$[\mathrm{s} / \mathrm{Fe}]$} \\
\hline 08544 & -0.3 & 0.0 & 0.0 & +0.2 & -0.2 & $/$ & +0.4 & +0.5 & +1.0 & +0.9 & $<-0.3$ \\
09060 & -0.7 & -0.7 & -0.2 & $/$ & $/$ & $/$ & +0.1 & -0.2 & 0.0 & +0.2 & +0.1 \\
09144 & -0.3 & 0.0 & 0.0 & +0.5 & -0.2 & 0.3 & $/$ & +0.6 & +1.3 & $/$ & $<-1.1$ \\
09538 & -0.6 & -0.6 & +0.4 & $/$ & +0.9 & 0.1 & -0.1 & +0.1 & +0.3 & +0.1 & $<-0.5$ \\
15469 & 0.0 & 0.2 & +0.3 & +0.6 & 0.0 & 0.9 & +0.3 & +0.9 & +2.1 & +1.8 & -0.6 \\
16230 & -0.7 & -0.4 & +0.2 & +0.2 & +0.3 & 0.4 & +0.3 & +0.4 & +1.1 & +1.0 & -0.4 \\
17038 & -1.5 & $/$ & +1.8 & $/$ & $/$ & $/$ & +0.3 & $/$ & $/$ & $/$ & -0.4 \\
17233 & -1.0 & -0.2 & +0.8 & +1.2 & +0.7 & 0.6 & +0.7 & +1.5 & +1.8 & +1.4 & $<-0.3$ \\
17243 & 0.0 & 0.2 & -0.2 & -0.2 & -0.1 & 0.4 & +0.2 & +0.2 & +0.6 & +0.8 & -1.4 \\
19125 & -0.3 & 0.1 & +0.5 & $/$ & +0.5 & 0.5 & +0.4 & +0.8 & $>2.6$ & $>2.3$ & $<-0.3$ \\
19157 & +0.1 & +0.1 & 0.0 & $/$ & +0.2 & 0.3 & $/$ & +0.4 & +0.4 & $/$ & $/$ \\
20056 & -0.3 & -0.2 & +0.6 & +1.1 & +0.6 & 0.5 & +0.1 & +0.5 & +1.2 & +1.2 & -0.5 \\
\hline Subdwarf & -0.2 & -0.2 & 0.0 & 0.0 & +0.1 & 0.4 & 0.0 & 0.0 & 0.0 & 0.0 & 0.0 \\
\hline
\end{tabular}

on the model parameters, is almost nil on the scatter of the ion abundance.

To get an idea of the influence of these uncertainties on the derived absolute and relative abundances, we computed the abundances for IRAS 16230-3410, using models which differ in effective temperature by $250 \mathrm{~K}$, in $\log$ gravity by 0.5 and in microturbulent velocity by $1.0 \mathrm{~km} \mathrm{~s}^{-1}$, respectively, from the preferred model. These are then compared with the abundances of the preferred model. We have chosen IRAS 16230-3410 because of its temperature, lying somewhat in the middle of the temperature range of the programme stars, the high amount of elements, for which an abundance is derived, and the many lines used. For the relative abundances we compared the abundance of the singly ionised atoms and those of S I, C I, N I and $\mathrm{O}$ I (neutral atoms with high excitation lines) with that of Fe II. All other abundances (i.e. those of neutral atoms with low excitation lines) are compared with that of Fe I.

The variations of the absolute and the relative abundances are listed in Table 4. In general, the uncertainties on the relative abundances are smaller than those on the absolute abundances. The variation of the abundances, due to the uncertainty on the temperature, is on average larger for the absolute abundances than for the relative abundances.

The Si II and Ba II abundances are both determined from one strong line ( $E W=137$ and $148 \mathrm{~m} \AA$, respectively) and are very sensitive to the assumed microturbulent velocities $\left(\sigma_{\xi_{\mathrm{t}}}=0.26\right.$ and 0.33 , respectively for the absolute abundances with $1.0 \mathrm{~km} \mathrm{~s}^{-1}$ change in microturbulent velocity). These two abundances, together with that of N I are the most influenced by the uncertainties on the model parameters. For the other relative abundances the total uncertainty $\sigma_{\text {rel,tot }}$ is less than 0.2 dex.

\section{Abundances}

The derived abundances are shown in Tables 5-7. For all ions, the solar abundance, the condensation temperature, the number of lines, the mean equivalent width, the absolute abundance, the $\sigma$ of the line-to-line scatter and the abundance to iron are given.

The objects are discussed individually in the Appendix on the basis of the Tables 5-7 and Fig. 6.

\subsection{Abundance synopsis}

In this section we discuss the synopsis of the abundance results, listed in Table 8. Note that the values for IRAS 17243-4348 are from the spectrum, taken in 2000 , except for [s/Fe]. In this table we include also the abundances for an unevolved mildly metal-poor dwarf $([\mathrm{Fe} / \mathrm{H}]=-0.2)$ (Nissen et al. 1985). These abundances reflect the chemical composition of the ISM out of which the stars (with an initial metallicity of -0.2) were formed. Differences in abundances between the programme stars and these abundances are the consequence of the changes in the chemical composition of the photosphere during the star's evolution. However, conclusions are not easily drawn on the basis of this comparison. First of all, it is possible that the observed metallicity does not correspond with the initial abundance of the star, because the depletion process has lowered the Fe-abundance. Secondly, abundance changes can be due to two processes: the depletion process and/or nucleosynthesis processes in the deep layers of the star, followed by deep mixing.

\section{Metallicities}

The observed metallicities range between -1.5 and +0.1 . We estimated the initial metallicity $[\mathrm{Fe} / \mathrm{H}]_{0}$ on the basis of the $\mathrm{Zn}$-abundance, if this abundance was believed to be unaffected. For the initial metallicity determination we made use of the relation: $[\mathrm{Zn} / \mathrm{Fe}]=+0.04$ from Sneden et al. (1991). Only for IRAS 17038-4815 and IRAS 17233-4330 $([\mathrm{Zn} / \mathrm{Fe}]=+0.7)$, was the metallicity greatly affected. Since for IRAS 17038-4815 also the $\mathrm{Zn}$-abundance is (probably) affected, it is difficult to trace the initial metallicity. 

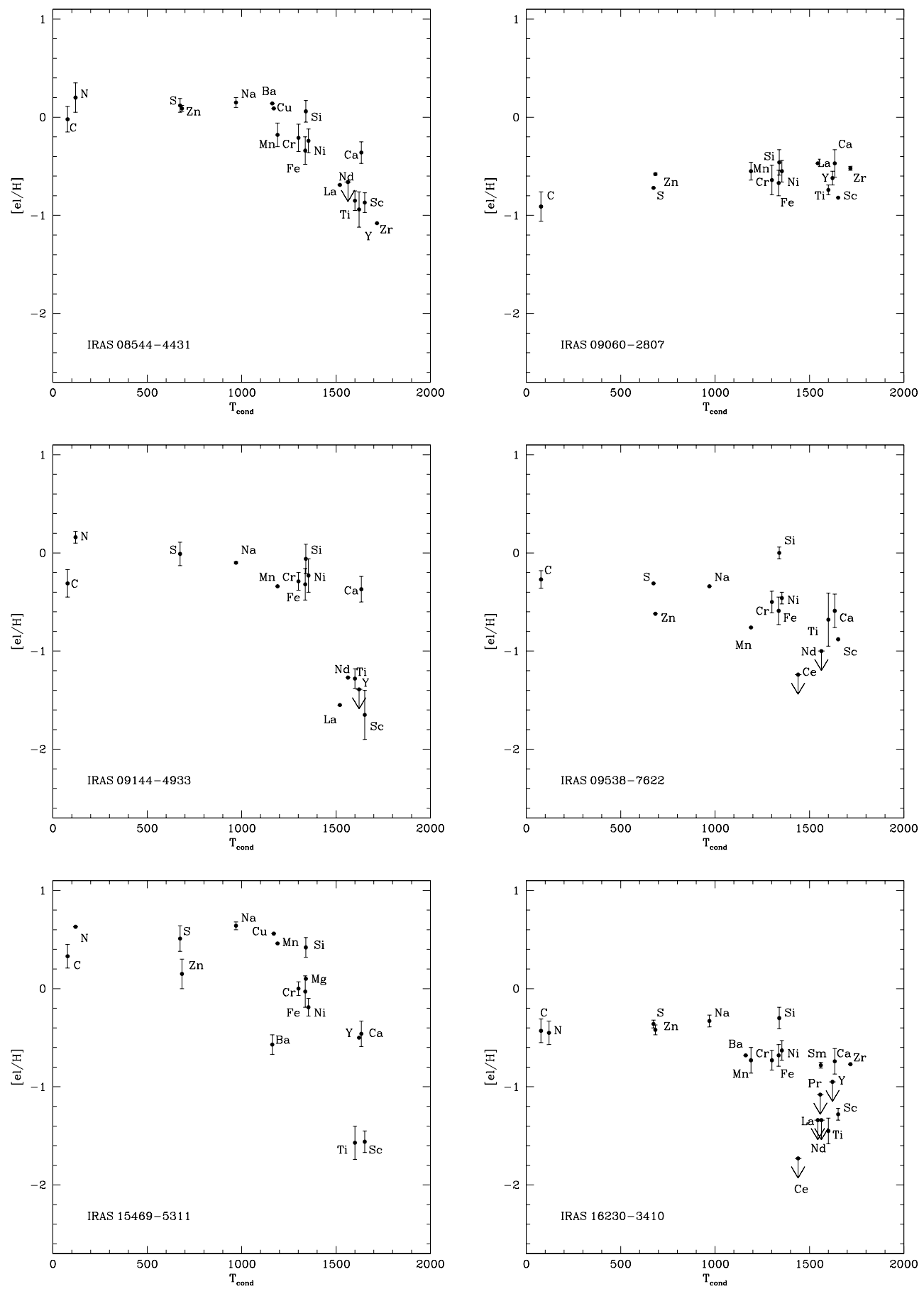

Fig. 6. The absolute abundances of the elements versus their condensation temperature for all the programme stars.

The estimated initial metallicity of IRAS $17233-4330$ is -0.2 . For IRAS 09060-2807 $([\mathrm{Fe} / \mathrm{H}]=-0.7)$, IRAS 09538-7622 $([\mathrm{Fe} / \mathrm{H}]=-0.6)$ and IRAS 19157-0247 $([\mathrm{Fe} / \mathrm{H}]=+0.1)$ the metallicity reflects the initial composition, because the abundances were not affected by a depletion process. For all the other stars, the influence of the depletion process on the metallicity was rather small.

\section{Depletion}

Based on the depletion process, discussed in the previous section, we can divide the programme stars in three groups.
In a first group, which consists of three objects, IRAS 09060-2807, IRAS 09538-7622 and IRAS 19157-0247, there is no evidence that a depletion process has taken place. IRAS 09538-7622 is a genuine RV Tauri star of spectroscopic group A.

The second group contains IRAS 08544-4431, IRAS 09144-4933， IRAS 15469-5311， IRAS 16230-3410, IRAS 17243-4348, IRAS 19125+0343 and IRAS 20056+1834. In these stars the elements with a condensation temperature up to about $1500 \mathrm{~K}$ (like $\mathrm{Fe}$ ) are not or only moderately depleted. Above that temperature most elements (the s-process elements, except for $\mathrm{Ba}, \mathrm{Ti}$ and $\mathrm{Sc}$ ) are clearly underabundant. 

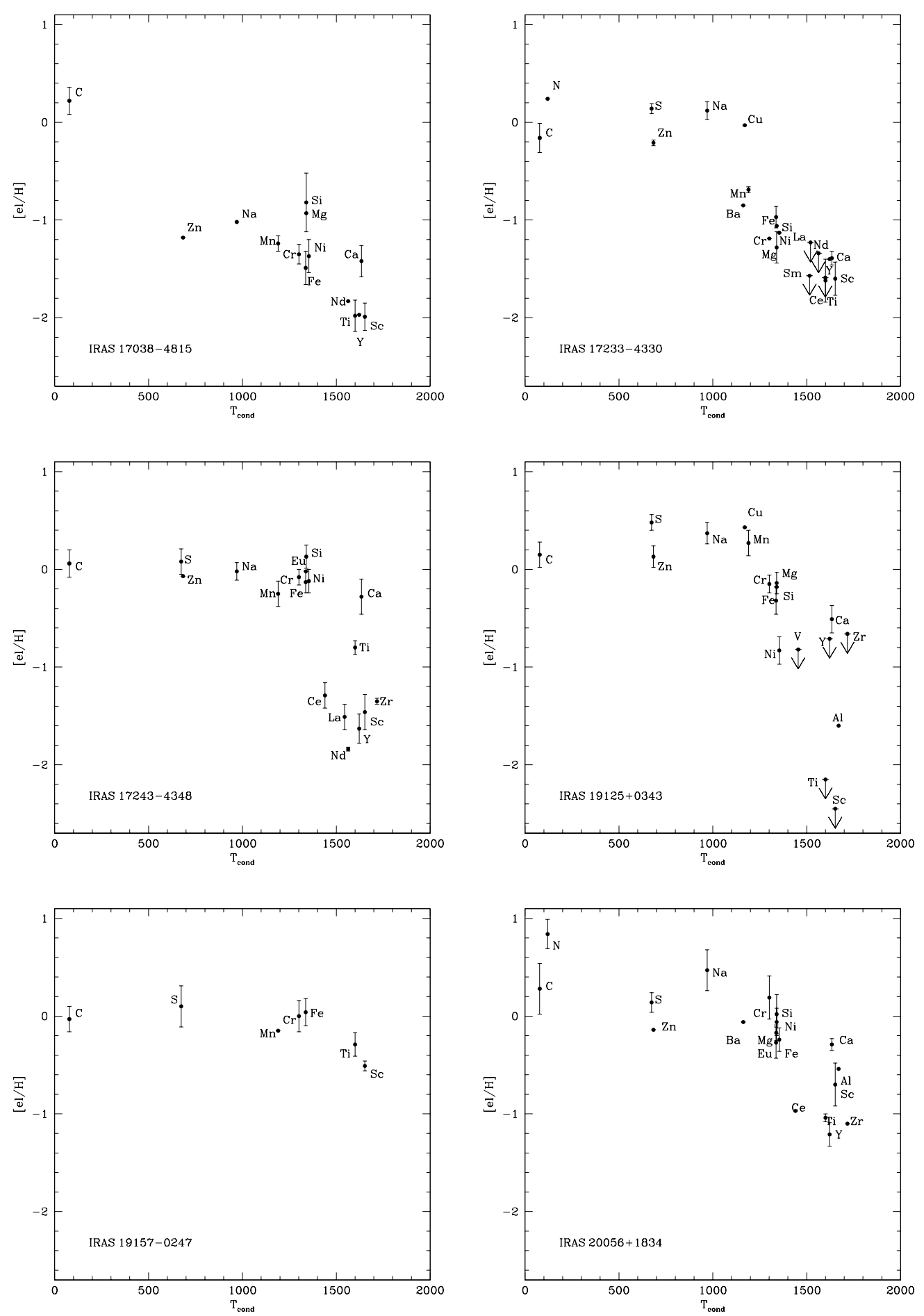

Fig. 6. continued.

We call this pattern in the abundance versus condensation temperature diagram, the "flat-steep" pattern in the following. This group contains also the genuine RV Tauri stars of spectroscopic group A, IRAS 16230-3410 and IRAS 17243-4348 (Lloyd Evans 1999, and in preparation).

The last group exists of the two genuine RV Tauri stars of spectroscopic group B: IRAS 17038-4815 and IRAS 17233-4330. A depletion process has strongly changed their abundances. For IRAS 17233-4330 the observed iron abundance differs from the estimated initial metallicity

by -0.8 . This was expected as all RV Tauri stars of spectroscopic group B, studied till now, are depleted.

\section{CNO abundances}

In all stars (except IRAS 17243-4348) [N/Fe] is positive, but only in IRAS 15469-5311, IRAS 17233-4330 and IRAS $20056+1834$ there is evidence that it is not only due to the decrease of the Fe-abundance but also the result of an enhancement in $\mathrm{N}$. The enhancement of the $\mathrm{N}$ abundance is most 
likely due to CNO-cycling and first dredge-up on the giant branch.

All $\mathrm{C} / \mathrm{O}$ ratios are smaller than 1 , so all stars are oxygen rich. The observed $\mathrm{C} / \mathrm{O}$ ratios are, within the errors, solar, except for IRAS 15469-5311 (C/O = 0.9) and IRAS 09538-7622 $(\mathrm{C} / \mathrm{O}=0.1)$. A higher than solar $\mathrm{C} / \mathrm{O}$ ratio can be caused by $\mathrm{C}$-nucleosynthesis on the AGB and subsequent dredge-up. Re-accretion of $\mathrm{CO}$ molecules will also change the $\mathrm{C} / \mathrm{O}$ ratio but it is unlikely that a $\mathrm{C} / \mathrm{O}$ ratio $>1$ can be expected: $\mathrm{CO}$ is the most abundant molecule after $\mathrm{H}_{2}$ and after efficient re-accretion $\mathrm{C} \sim \mathrm{O}$ is expected. The almost solar $\mathrm{C} / \mathrm{O}$ ratio for most of the stars can best be explained by the fact that no C-nucleosynthesis, followed by deep mixing, took place and that the depletion process operated only marginally in most of these stars. Together with the already mentioned small decrease of the iron abundance, also the amount of accreted material was low. Consequently, the effect on $\mathrm{C} / \mathrm{O}$ is small.

Since the depletion process has also been marginally active in IRAS 15469-5311, it is not likely that it is the cause of the high $\mathrm{C} / \mathrm{O}$ ratio. $\mathrm{C}$-nucleosynthesis on the $\mathrm{AGB}$ is mostly accompanied by the production of s-process elements. However, $\mathrm{Ba}$ and $\mathrm{Y}$ are the only s-process elements for which we could determine abundances, and both are underabundant.

The low C/O for IRAS 09538-7622 is probably caused by the high $\mathrm{O}$-abundance, which is based on only one line.

\section{Iron peak elements}

Both $\mathrm{Cr}$ and $\mathrm{Ni}$ have a condensation temperature close to that of Fe $\left(T_{\text {cond }}=1337 \mathrm{~K}\right)$, so the effects of the depletion process on the abundances of these elements, should be the same. This is confirmed in all stars in which the depletion process was active. Also in the other stars $\mathrm{Cr}$ and $\mathrm{Ni}$ follow the Fe abundance. $\mathrm{Zn}\left(T_{\text {cond }}=684 \mathrm{~K}\right)$ has a much lower condensation temperature than the other iron peak elements. For this reason $[\mathrm{Zn} / \mathrm{Fe}]$ is often used as a prime indicator for the depletion process. In the stars of the first group the low $[\mathrm{Zn} / \mathrm{Fe}]$ $(-0.1$ or +0.1$)$ points to the inactivity of the depletion process. In the second group $[\mathrm{Zn} / \mathrm{Fe}]$ varies between +0.1 and +0.4 , which is rather low. This can be explained as follows. In these stars elements with a condensation temperature up to $1500 \mathrm{~K}$, are slightly or not at all affected by the depletion process. Only elements with a condensation temperature higher than $1500 \mathrm{~K}$ are underabundant. As a consequence the "flat-steep" pattern in these stars is not traced by $[\mathrm{Zn} / \mathrm{Fe}]$ but more by $[\mathrm{Zn} / \mathrm{Ti}]$ or $[\mathrm{S} / \mathrm{Ti}]$ for instance. The low $[\mathrm{Zn} / \mathrm{Fe}]$ of +0.3 for IRAS $17038-4815$ is due to the fact that also the $\mathrm{Zn}$-abundance is likely affected.

\section{$\alpha$ elements}

Metal-poor unevolved dwarfs show $\alpha$-overabundances $([\alpha / \mathrm{Fe}]=+0.2$ for $[\mathrm{Fe} / \mathrm{H}]=-0.7$ Wheeler et al. 1989), reflecting the chemical history of our Galaxy. As the lowest initial metallicity in our programme stars is probably -0.7 (for IRAS 17038-4815 we can not estimate an initial metallicity), this effect is small. Moreover, the depletion process dilutes this effect, as $\alpha$ elements have different condensation temperatures: S: $674 \mathrm{~K}, \mathrm{Mg}: 1340 \mathrm{~K}, \mathrm{Si}: 1340 \mathrm{~K}, \mathrm{Ca}: 1634 \mathrm{~K}$, Ti: $1600 \mathrm{~K} .[\mathrm{S} / \alpha]$ is used to trace the depletion process. For stars of the second group, $[\mathrm{S} / \alpha]$ compares the abundance of $\mathrm{S}$ with that of two unaffected elements $(\mathrm{Mg}, \mathrm{Si})$ and two affected elements $(\mathrm{Ca}, \mathrm{Ti})$. Therefore it is not characteristic for this kinds of depletion. [S/Ti] seems therefore a better indicator for this "flat-steep" pattern.

\section{s-process elements}

We do not detect s-process enhancements in any of our objects. However, since s-process elements have a high $T_{\text {cond }}$ and the depletion process was active in most of the stars, possible s-process overabundance could be diluted. Nevertheless, if we compare the s-process abundances with the abundances of elements with the same condensation temperature, the s-process elements are not enriched. In this way we can conclude that there is no s-process evidence for AGB-nucleosynthesis and third dredge-up. In contrast, the s-process underabundances support in most stars the idea that a depletion process has taken place.

\section{Discussion}

\subsection{Abundance patterns}

The stars of our new sample have been selected on the basis of a large and broad IR excess. We limited our chemical abundance analysis to the stars with spectral type F and G. Clear chemical patterns which could be due to dredge-up processes during stellar evolution were not observed and we focus our discussion on depletion.

We observe three kind of depletion patterns. In the first group no depletion is observed, the metallicity corresponds to the initial metallicity. In the second group the picture is more complicated. Elements with a $T_{\text {cond }}$ below $1500 \mathrm{~K}$ are not or only slightly affected. Large underabundances are measured only for most elements with a $T_{\text {cond }}$ above $1500 \mathrm{~K}$. The third group contains two stars, in which the depletion process has considerably changed the chemical composition.

The uncertainties in the relative abundances, induced by the uncertainties in the model parameters, derived in Sect. 4.2, can not explain the low relative abundance of the elements with a high condensation temperature versus those with a low condensation temperature, observed in group 2 and 3 of the programme stars. Non-LTE effects are, however, not excluded and their impact on the abundances is difficult to assess. Such a non-LTE effect was put forward to explain s-process deficiencies in low-mass supergiants, which included type II Cepheids, RV Tauri stars and supergiants at a high latitude (Luck \& Bond 1989). They argued that in low mass variables a shock can be present, causing the production of Lyman-continuum photons. These photons lead to an overionisation of elements with a low second ionisation potential (IP) into the second-ionised state. This results in an underabundance for those elements in an LTE-analysis, which are mainly based on single ionised lines. All s-process elements together with $\mathrm{Sc}, \mathrm{Ca}$ and $\mathrm{Eu}$ have low 
second IPs $(<13.6 \mathrm{eV})$. Ti with a second IP of $13.58 \mathrm{eV}$ is right at the Lyman edge.

In Fig. 7 we plotted for three of our programme stars, IRAS 16230-3410, IRAS 17233-4330 and IRAS 09538-7622, the abundances of the elements versus their second IP. For IRAS 16230-3410 the strong underabundances are only observed for the elements with a second IP below $13.6 \mathrm{eV}$. However, for elements with a second IP below $13.6 \mathrm{eV}$, there is a large scatter in underabundances. This is also the case if one plots the abundances versus condensation temperature (see Fig. 6). This non-LTE effect can, however, not explain the small but significant $[\mathrm{Zn} / \mathrm{Fe}]=+0.3$. Both $\mathrm{Zn}$ and $\mathrm{Fe}$ have a second IP larger than $13.6 \mathrm{eV}$ and their abundances can not be affected by this non-LTE effect. The same is, to a larger extent, true for $[\mathrm{Zn} / \mathrm{Fe}$ ] in IRAS 17233-4330. Also the underabundances of $\mathrm{Mn}, \mathrm{Si}, \mathrm{Ni}, \mathrm{Cr}, \mathrm{Mg}$ can not be explained, since their second IP is higher than $13.6 \mathrm{eV}$.

If one looks only at the abundance patterns, this non-LTE effect can explain at least a part of the observed abundances for the programme stars with a flat-steep pattern. However, essential to affect the population of the different ionisation stages of the elements is of course the production of Lyman-continuum photons. The presence of a shock, creating these photons, is doubtful in many of our programme stars, in for example IRAS 15469-5311, for which the photometric variation is small ( $\Delta V=0.17$, Maas et al., in preparation). If this non-LTE effect would apply for IRAS 15469-5311, it is then surprising that it does not lead to underabundances in IRAS 09538-7622 (see Fig. 7), for which the peak-to-peak photometric variation in $V$ is high $(\Delta V=1.35$, Maas et al. in preparation) and shock caused line-deformation is observed in the cross-correlation profiles, which is not seen in IRAS 15469-5311 (Maas et al., in preparation).

Another way to discriminate between both explanations are the elements $\mathrm{Ba}$ and the r-process element Eu. They have both a low second ionization potential, but their condensation temperature $\left(T_{\text {cond }}=1162\right.$ and $1338 \mathrm{~K}$, respectively) is not as high as for the other s-process elements. In IRAS 08544-4431, IRAS 16230-3410, IRAS 17233-4330 and IRAS $20056+1834$, Ba scales with iron while it is underabundant in IRAS $15469-5311([\mathrm{Ba} / \mathrm{Fe}]=-0.6)$. Eu is solar in IRAS 17243-4348 and IRAS 20056+1834. Thus, the abundances of $\mathrm{Ba}$ and $\mathrm{Eu}$ are, in general, in favour of the depletion process. However, it must be stated that the $\mathrm{Ba}$ and $\mathrm{Eu}$ abundance are based on only one line and that the Eu line can be affected by hyper-fine splitting. Moreover, in the abundance versus condensation temperature diagram the scatter at a certain condensation temperature is sometimes also large.

Finally, we point to the fact that the underabundances found here are much larger than those found by Luck \& Bond (1989), which report underabundances, by a factor of about $2(0.3$ in logarithmic units). The underabundances for our stars attain values less than -2.1 for IRAS $19125+0343$.

We conclude that we found no observational evidence for this strong non-LTE effect to be active.

\subsection{Accretion scenarios}

Abundance anomalies resembling the chemical composition of the gas phase of the ISM are also observed in $\lambda$ Bootis. These are stars with effective temperature between $8000-10000 \mathrm{~K}$ and population I kinematics. They are often surrounded by the remains of their formation disc. The scale of the depletion in these stars is comparable with that in RV Tauri stars of spectroscopic group B. Bond (1991) proposed that selective removal of metals from the photosphere through grain formation and mass loss could account for the observed abundance anomalies in the strongly depleted post-AGB stars. The photosphere is, however, too hot for dust formation, so the gas-dust separation has to take place in the CS environment. Mathis \& Lamers (1992) pointed out two different possible accretion scenarios and Waters et al. (1992) stressed that the most likely place for the depletion process to take place is a stationary disc. In this scenario, the gas falls back on the star at a low accretion rate. The radiation pressure exerts an outward force on the dust and keeps it in the disc; the gas is, in contrast, not hampered by the radiation pressure under the condition that the density is low enough so that the gas is not dragged along with the dust. The observational evidence for the scenario of Waters et al. (1992) at that time were the discs surrounding the $\lambda$ Boo stars and the binarity of the two peculiar post-AGB binaries HR 4049 and $\mathrm{BD}+39^{\circ} 4926$. In the latter systems it is likely a disc has formed during mass-transfer at the AGB. Later on, the finding that all severely depleted post-AGB stars are binaries (Van Winckel et al. 1995) and the observed dust discs around some of them (Waelkens et al. 1991a; Roddier et al. 1995; Bond et al. 1997), enlarged the observational arguments for this scenario. This scenario became less clear after the detection of many more depleted systems (Giridhar et al. 2000, 1994, 1998; Gonzalez \& Lambert 1997; Gonzalez \& Wallerstein 1996; Gonzalez et al. 1997b; Van Winckel et al. 1998). Giridhar et al. (2000) considers two possible sites for the dust formation and dust-gas separation in RV Tauri stars: the dusty stellar wind in a single star or a dusty circumbinary disc.

Different kinds of data revealed that the prototypical RV Tauri star AC Her strengthens the link between the RV Tauri stars and the strongly depleted post-AGB stars and, as a consequence, the evidence for a circumbinary disc to be the most likely place for the gas-dust separation. Radial velocity monitoring shows that AC Her is a spectroscopic binary. Its photosphere is depleted and the SED of AC Her points to the presence of hot as well as cold dust (Van Winckel et al. 1998). Moreover, ISO-SWS spectra threw light on the oxygen rich nature of CS material of which a high amount is crystalline (Molster et al. 1999). Also the similarity with the ISO-SWS spectrum of the comet Hale-Bopp is striking. The CO-emission profile is different from that observed for post-AGB stars with an outflow (Bujarrabal et al. 1988). Last but not least, the disc around ACHer is resolved in the mid-IR (Jura et al. 2000). Recent observations could, however, not confirm this (Biller et al. 2003).

In this paper we analysed the chemical composition of stars with spectral type F and G, which were selected on the basis of a large IR excess: the object is surrounded by hot and cold dust. 
The most probable geometry of the dust around a post-AGB star with spectral type $F$ and $G$ is a disc. This work reveals that a high fraction ( 9 out of 12 or $75 \%$ ) of the sample shows the signature of depletion, indicating that a disc is a preferential site for the depletion process to take place. This strengthens the scenario of Waters et al. (1992). However, a disc is clearly not a sufficient condition for the dust-gas separation to take place, since three objects are not affected. In 89 Her there is also evidence for a disc (Waters et al. 1993), but no depletion is observed (Luck et al. 1990).

Dominik et al. (2003) outline that grain settling in the disc may play an essential role for accretion to take place. The larger grains will eventually settle in the midplane of the disc. The gas will then have a higher scale height than the dust. The dust free gas layers cease to experience an outward drag by the dust and, subsequently, fall back on the star.

Since the temperature in the disc decreases with increasing radius, at the inner radius of the disc only the elements with a high condensation temperature are caught in grains, given that the inner temperature of the disc is high. This could possibly give an explanation for the "flat-steep" depletion pattern observed in group 2 of the programme stars. When grains settle in the midplane, gas falls back on the star. This gas contains all elements except those with the highest condensation temperature. The accretion of this gas gives rise to the flat-steep pattern.

\subsection{Depletion and RV Tauri stars}

In what follows we compare our results concerning the observed chemical patterns with what is known from the chemical composition of RV Tauri stars and other depleted objects.

Table 9 shows the abundances for all analysed RV Tauri stars, the stars analysed in this paper, the strongly and moderately depleted post-AGB binaries, the type II Cepheid ST Pup and $\lambda$ Boo.

Giridhar et al. (2000) studied the chemical abundances of the RV Tauri stars and clearly established with this last paper in a series that depletion is widely observed in RV Tauri stars and is not just limited to a select club of peculiar objects (i.e. the severely depleted post-AGB binaries). In this publication nine of the twelve studied objects are affected by the depletion process. In this way, we subscribe that the depletion process is a widespread phenomenon.

Giridhar et al. (2000) pointed out a difference in chemical composition between the spectroscopic classes: the RV Tauri stars of spectroscopic group A are not or are only moderately affected, the stars of group B are severely affected and those of group $\mathrm{C}$ turn out to be immune for the depletion process.

In our sample, five genuine RV Tauri stars are present. In the stars of group A IRAS 16230-3410 and IRAS 17243-4348 strong underabundances are observed only for elements with a condensation temperature above $1500 \mathrm{~K}$, while no underabundances are observed in IRAS 09538-7622. The chemical compositions of the group B stars IRAS 17038-4815 and IRAS 17233-4330 shows signatures of severe depletion. These findings confirm their result.
Another of their results is that not only the scale of the depletion differs from star to star, but also the pattern in the abundance versus condensation temperature diagram. The RV Tauri stars were divided in several subgroups, according to the pattern observed. The "flat-steep" pattern we found in a large number of stars, is very similar to the one observed in R Scuti. DY Aql and CE Vir are possible members of this subgroup of R Scuti, but additional element abundances are needed. In the abundance synopsis of our stars we concluded that the abundance ratio [S/Ti] is a good tracer of the "flat-steep" pattern.

The difference in effective temperature between the RV Tauri stars of groups A and B is, according to Giridhar et al. (2000), the reason why stars of group B are heavily affected and stars of group A only moderately or not at all. The lower effective temperature of RV Tauri stars of group A results in a deeper convective envelope and dilutes the possible abundance anomalies. However, it is then not clear why some elements like $\mathrm{Ti}$ and $\mathrm{Sc}$ in some stars of group $\mathrm{A}$ are as underabundant as in RV Tauri stars of group B (see Table 9). The stars IRAS 08544-4431, IRAS 15469-5311 and IRAS 19125+0343, which show the "flat-steep" depletion pattern, are, on the basis of this, chemically similar to some RV Tauri stars of group A. However, with their $T_{\text {eff }}$ of $7250 \mathrm{~K}, 7500 \mathrm{~K}$ and $7750 \mathrm{~K}$ they are clearly hotter than the RV Tauri stars of group B, so convection is not a possible explanation for the lower depletion scale. The ongoing mass-loss, established by the P-Cygni profile of $\mathrm{H} \alpha$ in all three stars, maybe plays a role.

The minor role of the effective temperature on the strength of the depletion process is further established in Fig. 8. In this figure we plot for all depleted evolved stars the ratios $[\mathrm{Zn} / \mathrm{Fe}]$ and [S/Ti] versus $T_{\text {eff }}$. The open boxes correspond to stars with large pulsational amplitudes, while the full circles are stars with small pulsational amplitudes. For the former a mean effective temperature is difficult to determine due to its large variation during the pulsation cycle. No correlation is present between [ $\mathrm{Zn} / \mathrm{Fe}]$ and $T_{\text {eff }}$, while the variation in [S/Ti] around a $T_{\text {eff }}$ of $6000 \mathrm{~K}$ is 2 . The strongly depleted post-AGB binary HD 52961 illustrates this. With a $T_{\text {eff }}$ of $6000 \mathrm{~K}$ and a pulsation period of 70.8 days (Waelkens et al. 1991b; Fernie 1995), it is very similar to the RV Tauri stars. However, with a $[\mathrm{Zn} / \mathrm{Fe}]=+3.4$ it is much more depleted than any RV Tauri star (the highest $[\mathrm{Zn} / \mathrm{Fe}]=+2.0$ for $\mathrm{AD} \mathrm{Aql})$. A chemical analysis of the cooler objects in our sample in the future will allow us to check these findings.

For the unaffected RV Tauri stars of group C another reason for their immunity for the depletion process is suggested: their metallicity. The stars of group $\mathrm{C}$ have a low initial metallicity. The critical metallicity, below which the depletion process is not active, is estimated at $[\mathrm{Fe} / \mathrm{H}]=-1.0$ (Giridhar et al. 2000). How this low metallicity makes the depletion process inefficient is not yet clear. In the case of a storage site around a binary, a low metallicity can lead to a too slow dust formation so that the gas falls back on the star before the gas-dust separation has set in (Giridhar et al. 2000). We estimated for the objects listed in Table 9, the initial metallicity. For the RV Tauri stars we have taken the values from Giridhar et al. (2000). For the other objects in which depletion occurred, we estimated the initial Fe-metallicity on the 

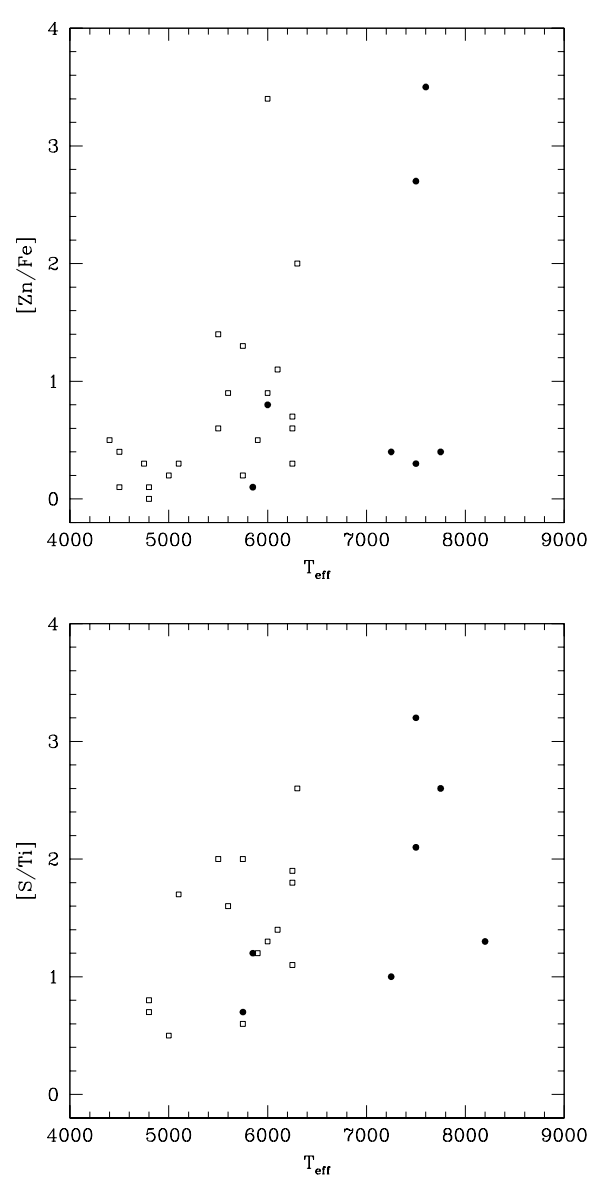

Fig. 8. The ratios $[\mathrm{Zn} / \mathrm{Fe}]$ and $[\mathrm{S} / \mathrm{Ti}]$ versus $T_{\text {eff }}$ for all depleted evolved stars, listed in Table 9. The open boxes indicate the stars with large pulsational amplitudes, while the full circles are stars with small pulsational amplitudes.

\subsection{Concluding remarks}

In this paper we performed a detailed analysis of high quality spectra of 12 stars with spectral type F and G of our new sample of RV Tauri like objects. Since nine of the twelve stars show depletion patterns, we confirm that depletion is a widespread phenomenon among post-AGB stars with a near IR excess. This depletion process takes place in the circumstellar environment. Elements with a high condensation temperature get locked in dust grains, while elements with a lower condensation temperature remain in the gas phase and are reaccreted. The radiation pressure keeps the dust from falling back on the star. We affirm that the efficiency of this process, together with the resulting abundance pattern, is different from star to star but that there is no correlation with the strength of the IR-excess (Giridhar et al. 2000). However, the presence of a broad IR-excess was the criterion on which the stars of our new sample were selected and in this paper we only analysed the stars with spectral type $F$ and G. For these stars the most obvious geometry for the surrounding dust is a circumbinary disc. The fact that a high fraction of $75 \%$ of the studied objects is depleted, indicates that the presence of a disc and the presence of depletion are linked. We find, however, that while a disc is a preferential site for the gas-dust separation to take place, its presence is not a sufficient condition.

In our following paper we will deal with the suspected binarity of the stars of our new sample and the relation with the presence of a disc and depletion.

Acknowledgements. The authors want to acknowledge the Geneva Observatory and its staff for the generous time allocation on the Swiss Euler telescope. T.M. acknowledges financial support from the Robert A Welch Foundation and from the Fund for Scientific Research of Flanders (FWO) under the grant G.0178.02. This paper is the publication of one chapter of the Ph.D. Thesis "A study of postAGB stars with a dusty disc" by T. Maas (2003), Het Instituut voor Sterrenkunde, KULeuven. The full Ph.D. Thesis is downloadable at http://www. ster.kuleuven.ac.be/pub/maas_phd/

\section{References}

Biémont, E., Hibbert, A., Godefroid, M., \& Vaeck, N. 1993, ApJ, 412, 431

Biémont, E., Hibbert, A., Godefroid, M., Vaeck, N., \& Fawcett, B. C. 1991, ApJ, 375, 818

Biller, B., Close, L., Potter, D., et al. 2003, in Asymmetric Planetary Nebulae III, 0

Bond, H. E. 1991, in Evolution of Stars: the Photospheric Abundance Connection, IAU Symp., 145, 341

Bond, H. E., Fulton, L. K., Schaefer, K. G., Ciardullo, R., \& Sipior, M. 1997, in Planetary Nebulae, IAU Symp., 180, 211

Bond, H. E., \& Luck, R. E. 1987, ApJ, 312, 203

Bujarrabal, V., Bachiller, R., Alcolea, J., \& Martin-Pintado, J. 1988, A\&A, 206, L17

Dominik, C., Dullemond, C. P., Cami, J., \& van Winckel, H. 2003, A\&A, 397, 595

Fernie, J. D. 1995, AJ, 110, 3010

Gehrz, R. D. 1972, ApJ, 178, 715

Giridhar, S., Lambert, D. L., \& Gonzalez, G. 1998, ApJ, 509, 366

Giridhar, S., Lambert, D. L., \& Gonzalez, G. 2000, ApJ, 531, 521 and IRAS 17233-4330). 
Giridhar, S., Rao, N. K., \& Lambert, D. L. 1990, The Observatory, 110,120

Giridhar, S., Rao, N. K., \& Lambert, D. L. 1992, ApA, 13, 307

Giridhar, S., Rao, N. K., \& Lambert, D. L. 1994, ApJ, 437, 476

Gonzalez, G., \& Lambert, D. L. 1997, AJ, 114, 341

Gonzalez, G., Lambert, D. L., \& Giridhar, S. 1997a, ApJ, 481, 452

Gonzalez, G., Lambert, D. L., \& Giridhar, S. 1997b, ApJ, 479, 427

Gonzalez, G., \& Wallerstein, G. 1996, MNRAS, 280, 515

Grevesse, N., \& Sauval, A. J. 1998, Space Sci. Rev., 85, 161

Herbig, G. H. 1970, ApJ, 162, 557

Hibbert, A., Biémont, E., Godefroid, M., \& Vaeck, N. 1991, A\&AS, 88,505

Holweger, H. 2001, in Joint SOHO/ACE workshop Solar and Galactic Composition, ed. R. F. Wimmer-Schweingruber, AIP Conf. Proc., 598,23

Jura, M. 1986, ApJ, 309, 732

Jura, M., Chen, C., \& Werner, M. W. 2000, ApJ, 541, 264

Kodaira, K. 1973, A\&A, 22, 273

Kupka, F., Piskunov, N., Ryabchikova, T. A., Stempels, H. C., \& Weiss, W. W. 1999, A\&AS, 138, 119

Kurucz, R. 1993, ATLAS9 Stellar Atmosphere Programs and 2 km/s grid, Kurucz CD-ROM No. 13, Cambridge, Mass.: Smithsonian Astrophysical Observatory, 13

Lloyd Evans, T. 1985, MNRAS, 217, 493

Lloyd Evans, T. 1997, Ap\&SS, 251, 239

Lloyd Evans, T. 1999, in Asymptotic Giant Branch Stars, ed. T. Le Bertre, A. Lèbre, \& C. Waelkens, IAU Symp., 191, 453

Lodders, K., \& Fegley, B. 1988, The Planetary Scientist's Companion (Oxford University Press), 83

Luck, R. E., \& Bond, H. E. 1984, ApJ, 279, 729

Luck, R. E., \& Bond, H. E. 1989, ApJ, 342, 476

Luck, R. E., Bond, H. E., \& Lambert, D. L. 1990, ApJ, 357, 188

Maas, T., Van Winckel, H., Lloyd Evans, T., et al. 2003, A\&A, 405, 271 (Paper I)

Maas, T., Van Winckel, H., \& Waelkens, C. 2002, A\&A, 386, 504

Mathis, J. S., \& Lamers, H. J. G. L. M. 1992, A\&A, 259, L39

Menzies, J. W., \& Whitelock, P. A. 1988, MNRAS, 233, 697
Molster, F. J., Waters, L. B. F. M., Trams, N. R., et al. 1999, A\&A, 350,163

Nissen, P. E., Edvardsson, B., \& Gustafsson, B. 1985, in Production and distribution of $\mathrm{C}, \mathrm{N}, \mathrm{O}$ elements, ESO Conference and Workshop Proceedings, held in Garching/Munich, May 13-15, 1985, Garching: European Southern Observatory (ESO), ed. I. J. Danziger, F. Mateucci, \& K. Kjär., 131

Preston, G. W., Krzeminski, W., Smak, J., \& Williams, J. A. 1963, ApJ, 137, 401

Rao, N. K., Goswami, A., \& Lambert, D. L. 2002, MNRAS, 334, 129

Raveendran, A. V. 1989, MNRAS, 238, 945

Roddier, F., Roddier, C., Graves, J. E., \& Northcott, M. J. 1995, ApJ, 443, 249

Sneden, C., Gratton, R. G., \& Crocker, D. A. 1991, A\&A, 246, 354

Stephenson, C. B. 1978, Information Bulletin on Variable Stars, 1453 , 1

Takeda, Y., Parthasarathy, M., Aoki, W., et al. 2002, PASJ, 54, 765

Van Winckel, H. 1995, The chemical composition of optically bright post-AGB stars (Ph.D. Thesis)

Van Winckel, H., Mathis, J. S., \& Waelkens, C. 1992, Nature, 356, 500

Van Winckel, H., \& Reyniers, M. 2000, A\&A, 354, 135

Van Winckel, H., Waelkens, C., \& Waters, L. B. F. M. 1995, A\&A, 293, L25

Van Winckel, H., Waelkens, C., Waters, L. B. F. M., et al. 1998, A\&A, 336, L17

Venn, K. A., \& Lambert, D. L. 1990, ApJ, 363, 234

Waelkens, C., Lamers, H. J. G. L. M., Waters, L. B. F. M., et al. 1991a, A\&A, 242, 433

Waelkens, C., Van Winckel, H., Bogaert, E., \& Trams, N. R. 1991b, A\&A, 251, 495

Waelkens, C., Van Winckel, H., Waters, L. B. F. M., \& Bakker, E. J. 1996, A\&A, 314, L17

Waters, L. B. F. M., Trams, N. R., \& Waelkens, C. 1992, A\&A, 262, L37

Waters, L. B. F. M., Waelkens, C., Mayor, M., \& Trams, N. R. 1993, A\&A, 269, 242

Wheeler, J. C., Sneden, C., \& Truran, J. W. 1989, ARA\&A, 27, 279 
T. Maas et al.: Depletion in post-AGB stars with a dusty disc. II., Online Material $p 1$

\section{Online Material}


T. Maas et al.: Depletion in post-AGB stars with a dusty disc. II., Online Material p 2

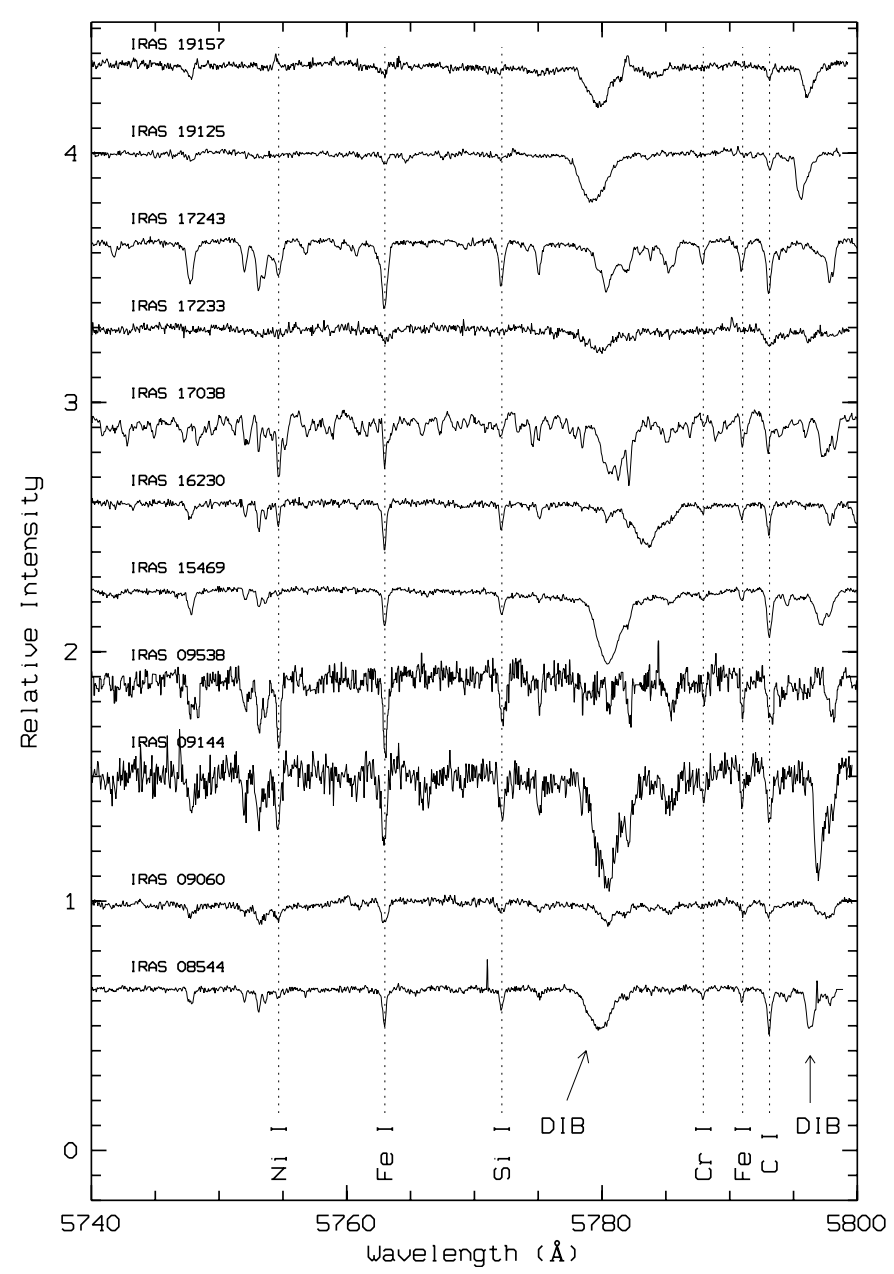

Fig. 3. The spectral domain between 5740-5800 $\AA$ with the Diffuse Interstellar Bands around $5780 \AA$ and $5797 \AA$. Note that we aligned the spectra on the photospheric lines.

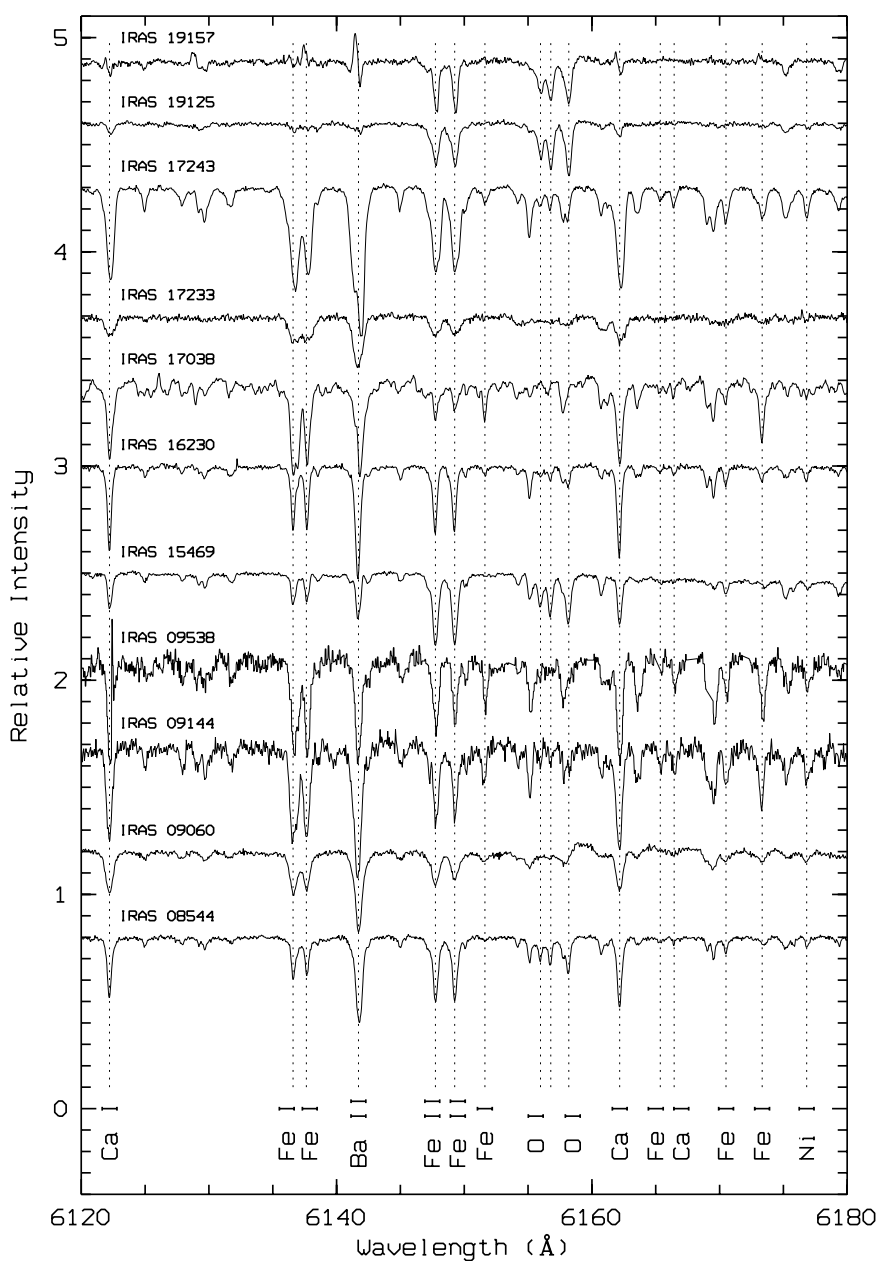

Fig. 4. The spectral domain between 6120-6180 $\mathrm{A}$. 
T. Maas et al.: Depletion in post-AGB stars with a dusty disc. II., Online Material p 3

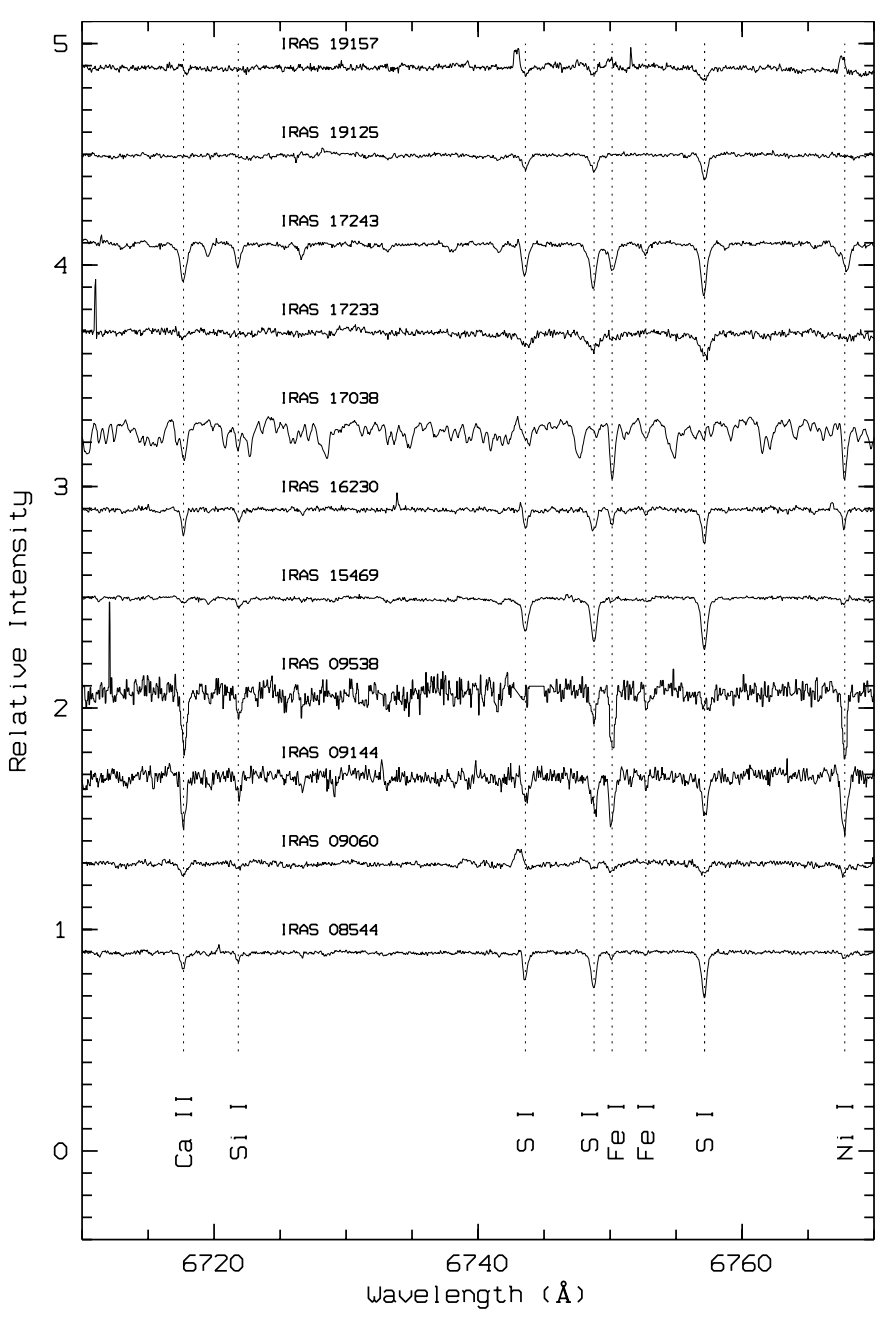

Fig. 5. The spectral domain between $6710-6770 \AA$ with the S-triplet at 6743,6749 and $6757 \AA$.

Table 2. Overview of the data. The date, the UT and the $S / N$ (measured around $5800 \AA$ ) of the analysed spectra are shown. All spectra were taken with the FEROS spectrograph, except for IRAS 09538-7622, for which we used a CORALIE spectrum.

\begin{tabular}{lllr}
\hline \hline IRAS & Date & UT & $S / N$ \\
\hline $08544-4431$ & $25 / 01 / 1999$ & $6: 20$ & 150 \\
$09060-2807$ & $23 / 03 / 2000$ & $0: 11$ & 140 \\
$09144-4933$ & $24 / 03 / 2000$ & $0: 31$ & 30 \\
$09538-7622$ & $05 / 12 / 1998$ & $7: 58$ & 40 \\
$15469-5311$ & $23 / 03 / 2000$ & $6: 26$ & 180 \\
& $27 / 06 / 2001$ & $2: 22$ & 140 \\
$16230-3410$ & $24 / 03 / 2000$ & $6: 00$ & 160 \\
$17038-4815$ & $24 / 03 / 2000$ & $8: 07$ & 160 \\
$17233-4330$ & $23 / 03 / 2000$ & $8: 13$ & 110 \\
$17243-4348$ & $24 / 03 / 2000$ & $9: 12$ & 140 \\
& $28 / 06 / 2001$ & $4: 19$ & 120 \\
$19125+0343$ & $28 / 06 / 2001$ & $7: 18$ & 190 \\
$19157-0247$ & $28 / 06 / 2001$ & $5: 52$ & 110 \\
\hline
\end{tabular}

\section{Appendix A: Individual objects}

The objects are now discussed individually on the basis of the Tables 5-7 and Fig 6. We look for chemical patterns
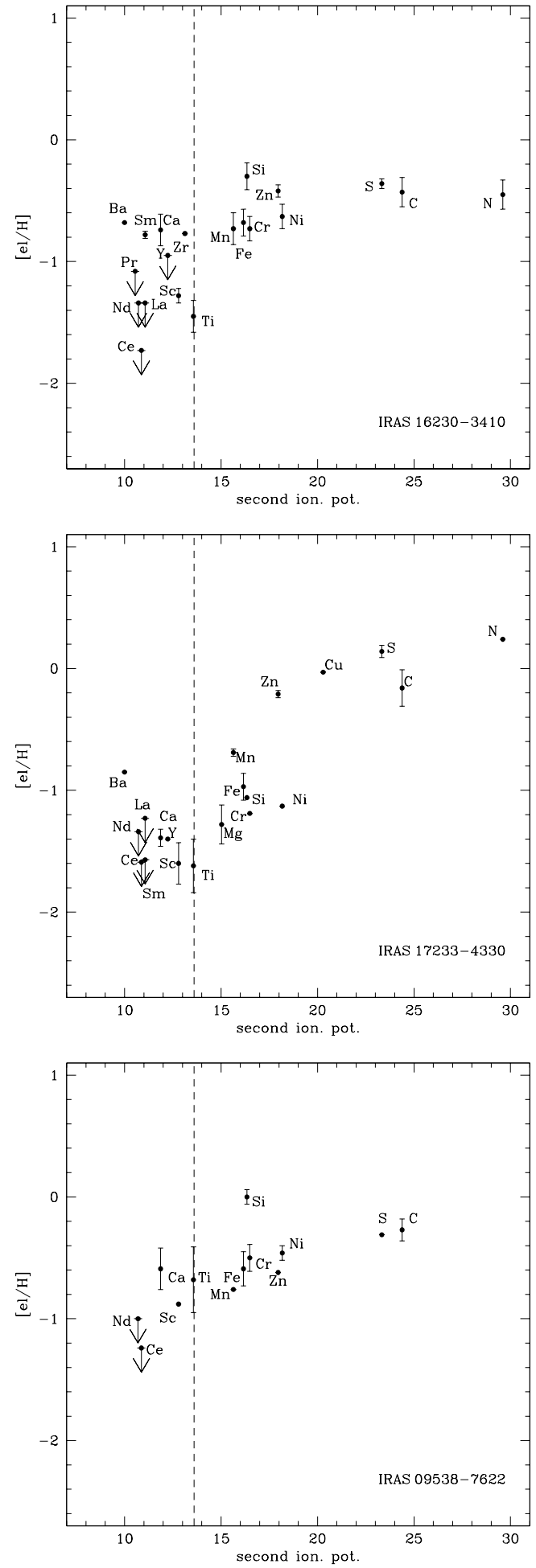

Fig. 7. The abundances of the elements versus their second ionisation potential for IRAS 16230-3410 $(\Delta V=0.46)$, IRAS 17233-4330 $(\Delta V=0.99)$ and IRAS 09538-7622 $(\Delta V=1.35)$ (Maas et al., in preparation). The dashed line represents the Lyman limit.

determined by dredge-up processes from the stellar interior. Alternatively, we look for chemical signatures pointing to depletion. A correlation between the abundance and the condensation temperature is taken as evidence for a dust-gas separation. However, even if a depletion process is the main effect 
Table 4. The influence of the uncertainties in the model parameters on the absolute and the relative abundances. For each ion, the absolute abundance is shown in the second column for the model of IRAS 16230-3410: $T_{\text {eff }}=6250 \mathrm{~K}, \log g=1.0, \xi_{t}=4.0 \mathrm{~km} \mathrm{~s}^{-1}$. The following three colums show the variation of the absolute abundance due to a decrease of $250 \mathrm{~K}$ in effective temperature $\left(\sigma_{T_{\text {eff }}}\right)$, due to a decrease of 0.5 in $\log g\left(\sigma_{g}\right)$ and due to a decrease of $1.0 \mathrm{~km} \mathrm{~s}^{-1}$ in microturbulent velocity $\left(\sigma_{\xi_{t}}\right)$, respectively. The sixth column shows the total variation of the absolute abundance $\sigma_{\text {abs,tot }}: \sigma_{\text {tot }}=\left(\sigma_{T_{\text {eff }}}{ }^{2}+\sigma_{g}{ }^{2}+\sigma_{\xi_{t}}{ }^{2}\right)^{1 / 2}$. The next columns show the corresponding effects on the relative abundances. The relative abundance, the variations of the relative abundance due to the variation of $T_{\text {eff }}, \log g$ and $\xi_{t}$ and the total variation of the relative abundance $\left(\sigma_{\text {rel,tot }}\right)$ are shown, respectively.

\begin{tabular}{lcrrrrrrrrr}
\hline \hline ion & $\log \epsilon$ & $\Delta T_{\text {eff }}$ & $\Delta \log g$ & $\Delta \xi_{t}$ & $\sigma_{\text {abs,tot }}$ & [el/Fe] & $\Delta T_{\text {eff }}$ & $\Delta \log g$ & $\Delta \xi_{t}$ & $\sigma_{\text {rel,tot }}$ \\
& & -250 & -0.5 & -1.0 & & & -250 & -0.5 & -1.0 & \\
\hline C I & 8.14 & +0.06 & -0.08 & +0.05 & +0.11 & +0.25 & +0.10 & +0.06 & -0.07 & +0.13 \\
N I & 7.54 & +0.23 & -0.13 & +0.07 & +0.27 & +0.23 & +0.27 & +0.01 & -0.05 & +0.27 \\
O I & 8.53 & +0.15 & -0.12 & +0.03 & +0.19 & +0.35 & +0.17 & +0.02 & -0.09 & +0.19 \\
Na I & 6.00 & -0.13 & +0.05 & +0.01 & +0.14 & +0.35 & +0.05 & +0.00 & -0.10 & +0.11 \\
Si I & 7.33 & -0.11 & +0.06 & +0.04 & +0.13 & +0.47 & +0.07 & +0.01 & -0.07 & +0.10 \\
Si II & 7.15 & +0.12 & -0.13 & +0.26 & +0.31 & +0.29 & +0.16 & +0.01 & +0.14 & +0.21 \\
S I & 6.97 & -0.03 & -0.03 & +0.04 & +0.06 & +0.32 & +0.01 & +0.11 & -0.08 & +0.14 \\
Ca I & 5.62 & -0.16 & +0.06 & +0.10 & +0.20 & -0.06 & +0.02 & +0.11 & -0.01 & +0.11 \\
Sc II & 0.89 & -0.11 & -0.14 & +0.02 & +0.18 & -0.60 & -0.07 & +0.00 & -0.10 & +0.12 \\
Ti II & 3.57 & -0.09 & -0.14 & +0.09 & +0.19 & -0.77 & -0.05 & +0.00 & -0.03 & +0.06 \\
Cr I & 4.88 & -0.26 & -0.01 & +0.00 & +0.26 & -0.11 & -0.08 & -0.06 & -0.11 & +0.15 \\
Cr II & 5.00 & -0.07 & -0.19 & +0.07 & +0.21 & +0.01 & +0.01 & -0.05 & -0.05 & +0.07 \\
Mn I & 4.66 & -0.18 & +0.05 & +0.07 & +0.20 & -0.05 & +0.00 & +0.00 & -0.04 & +0.04 \\
Fe I & 6.83 & -0.18 & +0.05 & +0.11 & +0.22 & & & & & \\
Fe II & 6.83 & -0.04 & -0.14 & +0.12 & +0.19 & & & & & \\
Ni I & 5.62 & -0.16 & +0.05 & +0.05 & +0.17 & +0.05 & +0.02 & +0.00 & -0.06 & +0.06 \\
Zn I & 4.18 & -0.19 & +0.04 & +0.17 & +0.26 & +0.26 & +0.01 & -0.01 & +0.06 & +0.06 \\
Zr II & 1.83 & -0.09 & -0.13 & +0.02 & +0.16 & -0.09 & -0.05 & +0.01 & -0.10 & +0.11 \\
Ba II & 1.45 & -0.23 & -0.04 & +0.33 & +0.40 & +0.00 & -0.19 & +0.10 & +0.21 & +0.30 \\
Sm II & 0.23 & -0.17 & -0.10 & +0.01 & +0.20 & -0.10 & -0.13 & -0.04 & -0.11 & +0.17 \\
\hline
\end{tabular}

present in the abundances, we do not expect a tight correlation between the abundance and the condensation temperature $T_{\text {cond }}$, since it is unlikely that the dust formation near the star occurs in thermal equilibrium and since the composition is, in general, not solar (The condensation temperatures are computed using a solar abundance mix at a pressure of $10^{-4}$ bar in thermal equilibrium.). We will come back to the effects of gas-dust separation and dredge-up in the next section.

All stars, for which we performed a chemical analysis, are pulsating variables. In this analysis we assume that we can approximate the atmosphere of the star at a certain pulsational phase by a hydrostatic model. For a part of the programme stars, the photometric variation is small (Lloyd Evans 1999) and this is very likely a good assumption. For the stars with a larger pulsational amplitude, it should be checked that the results of the chemical analysis at different pulsational phases are consistent. For one object, IRAS 17243-4348 ( $\Delta V=0.69$, Maas et al. in preparation), we have two spectra, taken at different pulsational phases, at our disposal. Although the derived model parameters are very different (a $T_{\text {eff }}$ of $6250 \mathrm{~K}$ and a $\log g$ of 0.5 for one phase and a $T_{\text {eff }}$ of $5250 \mathrm{~K}$ and a $\log g$ of 0.0 for the other phase), the results for the chemical composition are in good agreement. Gonzalez et al. (1997a) performed a chemical analysis of a number of RV Tauri stars. Several stars were observed on more than one occasion.
Spectra with absorption lines showing line-doubling or remarkable asymmetries or with strong emission in $\mathrm{H} \beta$ were rejected for analysis. Spectra, not showing these characteristics represent the atmosphere at its stablest time. Their analysis of three spectra of SS Gem, obtained at three different pulsational phases, is consistent. The analysed spectra of our stars do not show line-splitting, severe line-asymmetries or emission at $\mathrm{H} \beta$.

\section{IRAS 08544-4431}

The abundances of this object, of which the composition is moderately changed by the depletion process, are thoroughly discussed in Maas et al. (2003).

\section{IRAS 09060-2807}

Figure 6 shows that the abundances are independent of the condensation temperature and that all elements scale with iron. No depletion process has altered the chemical composition and the derived metallicity of -0.7 reflects the initial composition, indicating that it is an older object than the other stars in the sample. The old nature of this object is supported by its high galactic latitude of $12.94 \mathrm{deg}$. For a star of this metallicity one expects a small overabundance of $\alpha$-elements $[\alpha / \mathrm{Fe}]=0.2$. For IRAS 09060-2807 we find 
Table 5. Abundances for programme stars. For all ions, the solar abundance, the condensation temperature, the number of lines, the mean equivalent width, the absolute abundance, the $\sigma$ of the line-to-line scatter and the abundance relative to iron are given. For the solar iron abundance we used the meteoric value of 7.51. For the solar C,N and O abundances we adopted resp. 8.57, 7.99 and 8.86 (C: Biémont et al. 1993; N: Hibbert et al. 1991; O: Biémont et al. 1991). For the solar Mg and Si abundances we adopt 7.54 (Holweger 2001); the other solar abundances are taken from Grevesse \& Sauval (1998). The dust condensation temperatures are from Lodders \& Fegley (1988) and references therein. Only for Si do we use a different condensation temperature than that given in Lodders \& Fegley (1988). We use a temperature of $1340 \mathrm{~K}$, which is the temperature at which $\mathrm{MgSiO}_{3}$ and $\mathrm{Mg}_{2} \mathrm{SiO}_{4}$, which contain most $\mathrm{Si}$, condense (Lodders \& Fegley 1988 ). The abundances shown for IRAS 15469-5311 are derived from the spectrum, which was taken in 2000. The analysis of the spectrum, which was taken in 2001 , leads to very similar abundances.

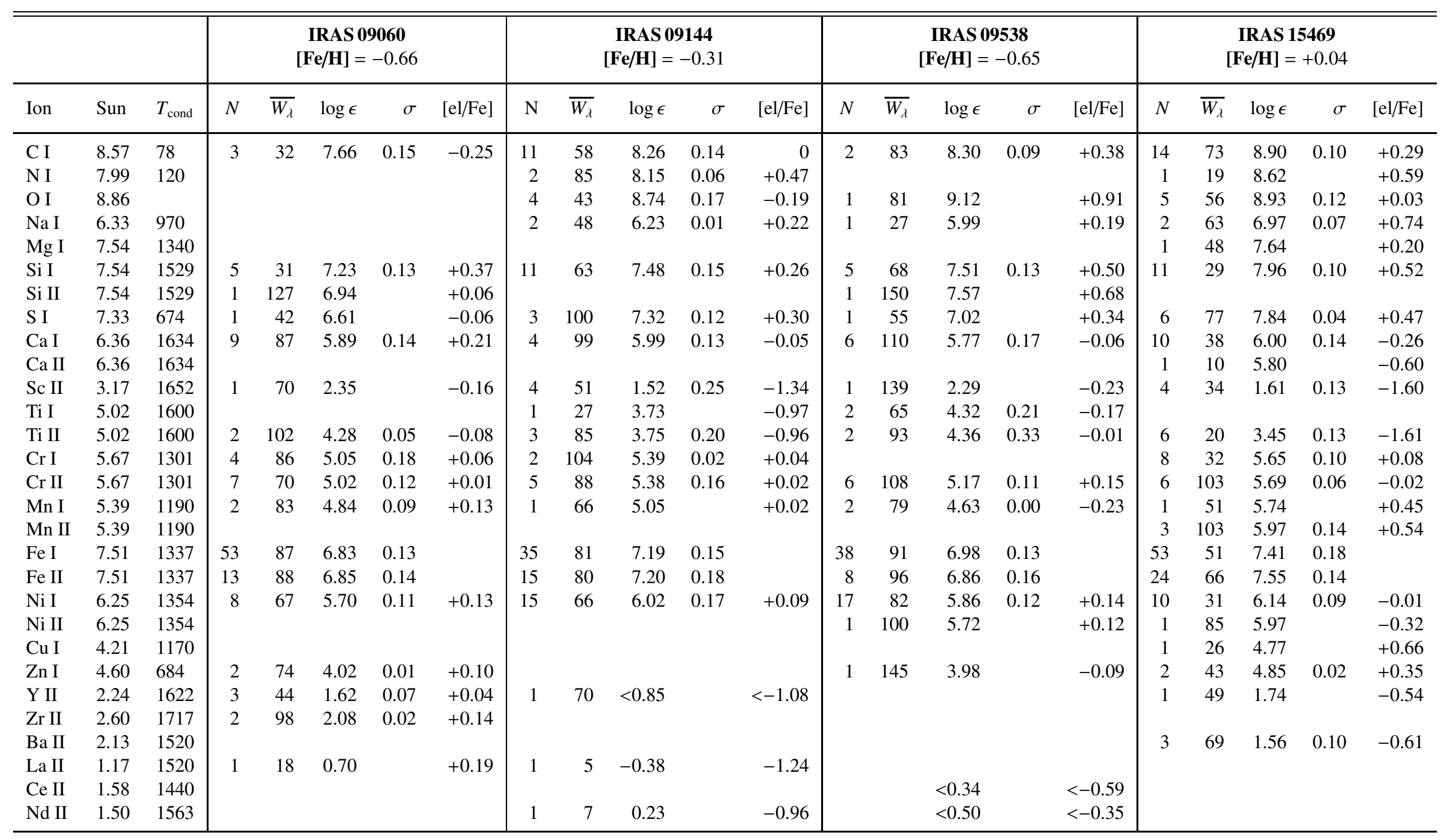


Table 6. Continuation of Table 5. Abundances for IRAS 16230-3410, IRAS 17038-4815 and IRAS 17233-4330.

\begin{tabular}{|c|c|c|c|c|c|c|c|c|c|c|c|c|c|c|c|c|c|}
\hline \multirow[b]{2}{*}{ Ion } & \multirow[b]{2}{*}{ Sun } & \multirow[b]{2}{*}{$T_{\text {cond }}$} & \multicolumn{5}{|c|}{$\begin{array}{c}\text { IRAS 16230 } \\
{[\mathrm{Fe} / \mathrm{H}]=-0.68}\end{array}$} & \multicolumn{5}{|c|}{$\begin{array}{c}\text { IRAS 17038 } \\
{[\mathrm{Fe} / \mathrm{H}]=-1.54}\end{array}$} & \multicolumn{5}{|c|}{$\begin{array}{c}\text { IRAS 17233 } \\
{[\text { Fe/H] }=-0.98}\end{array}$} \\
\hline & & & $N$ & $\overline{W_{\lambda}}$ & $\log \epsilon$ & $\sigma$ & {$[\mathrm{el} / \mathrm{Fe}]$} & $N$ & $\overline{W_{\lambda}}$ & $\log \epsilon$ & $\sigma$ & {$[\mathrm{el} / \mathrm{Fe}]$} & $N$ & $\overline{W_{\lambda}}$ & $\log \epsilon$ & $\sigma$ & {$[\mathrm{el} / \mathrm{Fe}]$} \\
\hline C I & 8.57 & 78 & 15 & 68 & 8.14 & 0.12 & +0.25 & 3 & 64 & 8.79 & 0.14 & +1.76 & 10 & 87 & 8.41 & 0.15 & +0.82 \\
\hline N I & 7.99 & 120 & 2 & 46 & 7.54 & 0.12 & +0.23 & & & & & & 1 & 25 & 8.23 & & +1.22 \\
\hline O I & 8.86 & & 3 & 30 & 8.53 & 0.10 & +0.35 & & & & & & 3 & 27 & 8.60 & 0.04 & +0.72 \\
\hline $\mathrm{Na} \mathrm{I}$ & 6.33 & 970 & 2 & 20 & 6.00 & 0.06 & +0.35 & 1 & 73 & 5.31 & & +0.43 & 2 & 108 & 6.45 & 0.09 & +1.05 \\
\hline Mg I & 7.54 & 1340 & & & & & & 1 & 100 & 6.61 & & +0.52 & & & & & \\
\hline Mg II & 7.54 & 1340 & & & & & & & & & & & 2 & 141 & 6.26 & 0.16 & -0.30 \\
\hline Si I & 7.54 & 1529 & 15 & 38 & 7.33 & 0.11 & +0.47 & 3 & 56 & 6.72 & 0.30 & +0.63 & & & & & \\
\hline Si II & 7.54 & 1529 & 1 & 137 & 7.15 & & +0.29 & & & & & & 1 & 97 & 6.48 & & -0.08 \\
\hline S I & 7.33 & 674 & 5 & 45 & 6.97 & 0.04 & +0.32 & & & & & & 4 & 108 & 7.47 & 0.05 & +1.12 \\
\hline $\mathrm{Ca} \mathrm{I}$ & 6.36 & 1634 & 16 & 70 & 5.62 & 0.13 & -0.06 & 7 & 94 & 4.94 & 0.16 & +0.03 & 5 & 79 & 4.97 & 0.07 & -0.46 \\
\hline Ca II & 6.36 & 1634 & & & & & & & & & & & & & & & \\
\hline Sc II & 3.17 & 1652 & 3 & 32 & 0.89 & 0.06 & -1.60 & 5 & 73 & 1.18 & 0.14 & -0.45 & 2 & 104 & 1.57 & 0.17 & -0.62 \\
\hline Ti I & 5.02 & 1600 & & & & & & & & & & & & & & & \\
\hline Ti II & 5.02 & 1600 & 21 & 73 & 3.57 & 0.13 & -0.77 & 7 & 92 & 3.04 & 0.16 & -0.44 & 3 & 48 & 3.40 & 0.22 & -0.64 \\
\hline Cr I & 5.67 & 1301 & 8 & 53 & 4.88 & 0.11 & -0.11 & 1 & 37 & 4.35 & & +0.13 & 1 & 78 & 4.47 & & -0.27 \\
\hline Cr II & 5.67 & 1301 & 13 & 82 & 5.00 & 0.10 & +0.01 & 3 & 66 & 4.30 & 0.19 & +0.17 & 5 & 82 & 4.48 & 0.16 & -0.21 \\
\hline Mn I & 5.39 & 1190 & 6 & 54 & 4.66 & 0.13 & -0.05 & 4 & 96 & 4.15 & 0.08 & +0.21 & 2 & 119 & 4.70 & 0.03 & +0.24 \\
\hline $\mathrm{Fe} \mathrm{I}$ & 7.51 & 1337 & 92 & 70 & 6.83 & 0.13 & & 33 & 79 & 6.06 & 0.20 & & 37 & 102 & 6.58 & 0.12 & \\
\hline $\mathrm{Fe}$ II & 7.51 & 1337 & 24 & 82 & 6.83 & 0.10 & & 12 & 101 & 5.97 & 0.15 & & 11 & 99 & 6.53 & 0.10 & \\
\hline Ni I & 6.25 & 1354 & 24 & 45 & 5.62 & 0.10 & +0.05 & 12 & 65 & 4.88 & 0.15 & +0.08 & 1 & 67 & 5.12 & & -0.20 \\
\hline $\mathrm{Cu} \mathrm{I}$ & 4.21 & 1170 & & & & & & & & & & & 1 & 90 & 4.18 & & +0.90 \\
\hline $\mathrm{Zn} \mathrm{I}$ & 4.60 & 684 & 2 & 100 & 4.18 & 0.05 & +0.26 & 1 & 150 & 3.42 & & +0.27 & 2 & 146 & 4.39 & 0.03 & +0.72 \\
\hline Y II & 2.24 & 1622 & & & $<1.29$ & & $<-0.27$ & 1 & 6 & 0.27 & & -0.43 & 1 & 9 & 0.84 & & -0.42 \\
\hline $\mathrm{Zr}$ II & 2.60 & 1717 & 1 & 20 & 1.83 & & -0.09 & & & & & & & & & & \\
\hline $\mathrm{Ba}$ II & 2.13 & 1520 & 1 & 148 & 1.45 & & 0.00 & & & & & & 1 & 134 & 1.28 & & +0.13 \\
\hline La II & 1.17 & 1520 & & & $<-0.17$ & & $<-0.66$ & & & & & & & & $<-0.06$ & & $<-0.25$ \\
\hline Ce II & 1.58 & 1440 & & & $<-0.15$ & & $<-1.05$ & & & & & & & & $<-0.01$ & & $<-0.61$ \\
\hline Pr II & 0.71 & 1557 & & & $<-0.37$ & & $<-0.40$ & & & & & & & & & & \\
\hline Nd II & 1.50 & 1563 & & & $<0.16$ & & $<-0.66$ & 1 & 39 & -0.33 & & -0.29 & & & $<0.16$ & & $<-0.36$ \\
\hline Sm II & 1.01 & 1560 & 2 & 14 & 0.23 & 0.03 & -0.10 & & & & & & & & $<-0.56$ & & $<-0.59$ \\
\hline
\end{tabular}


Table 7. Continuation of Table 5. Abundances for IRAS 17243-4348, IRAS 19125+0343 and IRAS 19157-0247 For IRAS 17243-4348 we show the abundances, derived from both spectra.

\begin{tabular}{|c|c|c|c|c|c|c|c|c|c|c|c|c|c|c|c|c|c|c|c|c|c|c|}
\hline \multirow[b]{2}{*}{ Ion } & \multirow[b]{2}{*}{ Sun } & \multirow[b]{2}{*}{$T_{\text {cond }}$} & \multicolumn{5}{|c|}{$\begin{array}{c}\text { IRAS 17243 } \\
24 / 03 / 2000 \\
{[\mathrm{Fe} / \mathrm{H}]=-0.05}\end{array}$} & \multicolumn{5}{|c|}{$\begin{array}{c}\text { IRAS 17243 } \\
28 / 06 / 2001 \\
{[\mathrm{Fe} / \mathrm{H}]=-0.10}\end{array}$} & \multicolumn{5}{|c|}{$\begin{array}{c}\text { IRAS } 19125 \\
{[\mathrm{Fe} / \mathrm{H}]=-0.35}\end{array}$} & \multicolumn{5}{|c|}{$\begin{array}{c}\text { IRAS } 19157 \\
{[\mathrm{Fe} / \mathrm{H}]=+0.07}\end{array}$} \\
\hline & & & $N$ & $\overline{W_{\lambda}}$ & $\log \epsilon$ & $\sigma$ & {$[\mathrm{el} / \mathrm{Fe}]$} & $N$ & $\overline{W_{\lambda}}$ & $\log \epsilon$ & $\sigma$ & {$[\mathrm{el} / \mathrm{Fe}]$} & $N$ & $\overline{W_{\lambda}}$ & $\log \epsilon$ & $\sigma$ & {$[\mathrm{el} / \mathrm{Fe}]$} & $N$ & $\overline{W_{\lambda}}$ & $\log \epsilon$ & $\sigma$ & {$[\mathrm{el} / \mathrm{Fe}]$} \\
\hline C I & 8.57 & 78 & 23 & 72 & 8.35 & 0.12 & -0.17 & 10 & 86 & 8.63 & 0.14 & 0.16 & 24 & 57 & 8.72 & 0.13 & +0.50 & 13 & 45 & 8.61 & 0.13 & -0.03 \\
\hline N I & 7.99 & 120 & 5 & 58 & 7.78 & 0.15 & -0.16 & & & & & & & & & & & & & & & \\
\hline O I & 8.86 & & 3 & 53 & 8.73 & 0.01 & -0.08 & & & & & & 8 & 76 & 9.06 & 0.12 & +0.55 & 5 & 85 & 9.15 & 0.12 & +0.22 \\
\hline $\mathrm{Na} \mathrm{I}$ & 6.33 & 970 & 2 & 54 & 6.57 & 0.00 & 0.26 & 2 & 77 & 6.31 & 0.09 & 0.14 & 3 & 35 & 6.70 & 0.11 & +0.66 & & & & & \\
\hline Mg I & 7.54 & 1340 & 1 & 137 & 7.65 & & 0.13 & & & & & & 1 & 125 & 7.51 & & +0.26 & & & & & \\
\hline Mg II & 7.54 & 1340 & & & & & & & & & & & 1 & 71 & 7.29 & & +0.10 & & & & & \\
\hline $\mathrm{Al} \mathrm{I}$ & 6.47 & 1670 & & & & & & & & & & & 1 & 57 & 4.87 & & -1.31 & & & & & \\
\hline $\mathrm{Si} I$ & 7.54 & 1529 & 16 & 68 & 7.81 & 0.12 & 0.29 & 11 & 101 & 7.67 & 0.12 & 0.29 & 1 & 9 & 7.36 & & +0.11 & & & & & \\
\hline Si II & 7.54 & 1529 & 1 & 137 & 7.92 & & 0.43 & & & & & & & & & & & & & & & \\
\hline S I & 7.33 & 674 & 6 & 88 & 7.37 & 0.09 & 0.09 & 6 & 91 & 7.41 & 0.13 & 0.18 & 6 & 37 & 7.81 & 0.08 & +0.83 & 3 & 30 & 7.50 & 0.21 & +0.10 \\
\hline $\mathrm{Ca} \mathrm{I}$ & 6.36 & 1634 & 9 & 91 & 6.19 & 0.10 & -0.15 & 5 & 113 & 6.08 & 0.18 & -0.12 & 3 & 27 & 5.85 & 0.14 & -0.22 & & & & & \\
\hline Sc II & 3.17 & 1652 & 1 & 119 & 2.06 & & -1.06 & 4 & 90 & 1.71 & 0.18 & -1.36 & & & $<0.72$ & & $<-2.10$ & 3 & 40 & 2.73 & 0.05 & -0.51 \\
\hline Ti I & 5.02 & 1600 & & & & & & 2 & 23 & 4.11 & 0.15 & -0.75 & & & & & & & & & & \\
\hline Cr I & 5.67 & 1301 & & & & & & 6 & 92 & 5.49 & 0.12 & -0.02 & 4 & 63 & 5.48 & 0.07 & +0.10 & & & & & \\
\hline Cr II & 5.67 & 1301 & 1 & 80 & 5.60 & & -0.02 & 2 & 87 & 5.50 & 0.05 & -0.07 & 16 & 81 & 5.56 & 0.12 & +0.24 & 10 & 95 & 5.74 & 0.16 & +0.00 \\
\hline Mn I & 5.39 & 1190 & 2 & 75 & 5.30 & 0.02 & -0.07 & 3 & 88 & 5.14 & 0.13 & -0.09 & 3 & 35 & 5.66 & 0.13 & +0.56 & 1 & 17 & 5.26 & & -0.15 \\
\hline $\mathrm{Fe} \mathrm{I}$ & 7.51 & 1337 & 53 & 66 & 7.49 & 0.12 & & 43 & 88 & 7.35 & 0.07 & & 45 & 47 & 7.22 & 0.16 & & 19 & 55 & 7.53 & 0.13 & \\
\hline $\mathrm{Fe}$ II & 7.51 & 1337 & 13 & 82 & 7.46 & 0.12 & & 13 & 78 & 7.41 & 0.16 & & 18 & 53 & 7.16 & 0.12 & & 23 & 71 & 7.58 & 0.15 & \\
\hline Ni I & 6.25 & 1354 & 25 & 71 & 6.26 & 0.11 & 0.03 & 16 & 111 & 6.13 & 0.12 & 0.04 & 1 & 12 & 5.56 & & -0.40 & & & & & \\
\hline Ni II & 6.25 & 1354 & & & & & & & & & & & 1 & 24 & 5.27 & & -0.63 & & & & & \\
\hline $\mathrm{Cu} \mathrm{I}$ & 4.21 & 1170 & 1 & 113 & 4.49 & & 0.30 & & & & & & 1 & 7 & 4.64 & & +0.72 & & & & & \\
\hline $\mathrm{Zn} \mathrm{I}$ & 4.60 & 684 & 1 & 65 & 4.82 & & 0.24 & 1 & 92 & 4.53 & & 0.09 & 2 & 24 & 4.73 & 0.11 & +0.42 & & & & & \\
\hline Y II & 2.24 & 1622 & 1 & 6 & 0.44 & & -1.75 & 3 & 49 & 0.61 & 0.15 & -1.53 & 1 & 17 & $<1.53$ & & $<-0.36$ & & & & & \\
\hline $\mathrm{Zr}$ II & 2.60 & 1717 & & & & & & 2 & 73 & 1.25 & 0.03 & -1.25 & 1 & 14 & $<1.94$ & & $<-0.31$ & & & & & \\
\hline La II & 1.17 & 1520 & & & & & & 2 & 9 & -0.34 & 0.13 & -1.41 & & & & & & & & & & \\
\hline Ce II & 1.58 & 1440 & & & & & & 3 & 66 & 0.29 & 0.13 & -1.19 & & & & & & & & & & \\
\hline Nd II & 1.50 & 1563 & & & & & & 2 & 7 & -0.34 & 0.02 & -1.74 & & & & & & & & & & \\
\hline Eu II & 0.51 & 1338 & 1 & 50 & 0.75 & & 0.29 & 1 & 85 & 0.49 & & 0.08 & & & & & & & & & & \\
\hline
\end{tabular}


Table 9. Abundances of the RV Tauri stars, our programme stars, the severely and mildly depleted post-AGB binaries, the Population II Cepheid ST Pup and $\lambda$ Boo. The second column refers to the spectroscopic class of the RV Tauri stars. We also included the $T_{\text {eff }}$. For the genuine RV Tauri stars we took the mean temperature, if more than one temperature was measured during the pulsation cycle.

\begin{tabular}{|c|c|c|c|c|c|c|c|c|c|}
\hline & Class & {$[\mathrm{S} / \mathrm{H}]$} & {$[\overline{\mathrm{Zn} / \mathrm{H}]}$} & {$[\overline{[S c / H]}$} & {$[\overline{\mathrm{TTi} / \mathrm{H}]}$} & {$[\overline{[F e} / \mathrm{H}]$} & $\overline{[\mathrm{Fe} / \mathrm{H}]_{0}}$ & 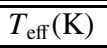 & Ref. \\
\hline DY Aql & $\mathrm{A}$ & & & -2.1 & & -1.0 & & 4250 & 1 \\
\hline TW Cam & A & 0.0 & -0.4 & -0.5 & -0.7 & -0.5 & -0.4 & 4800 & 2 \\
\hline SS Gem & A & -0.4 & 0.0 & -1.9 & -2.0 & -0.9 & -0.2 & 5600 & 1 \\
\hline U Mon & A & -0.1 & -0.6 & -1.0 & -0.6 & -0.8 & -0.5 & 5000 & 2 \\
\hline TT Oph & A & 0.0 & -0.8 & -1.1 & -0.8 & -0.8 & -0.8 & 4800 & 2 \\
\hline R Sct & A & & -0.3 & -1.4 & -0.4 & -0.4 & -0.3 & 4500 & 2 \\
\hline R Sge & A & 0.4 & -0.2 & -1.5 & -1.3 & -0.5 & 0.1 & 5100 & 3 \\
\hline RV Tau & A & & 0.0 & -0.3 & -0.5 & -0.4 & -0.4 & 4500 & 2 \\
\hline CE Vir & A & & -0.7 & -2.6 & -1.3 & -1.2 & -0.7 & 4400 & 1 \\
\hline AD Aql & B & 0.0 & -0.1 & -1.8 & -2.6 & -2.1 & -0.1 & 6300 & 4 \\
\hline UY Ara & B & 0.0 & -0.4 & -1.7 & & -1.0 & -0.3 & 5500 & 2 \\
\hline IW Car & B & 0.4 & -0.1 & & & & +0.2 & 6700 & 5 \\
\hline RU Cen & B & -0.7 & -1.0 & -1.9 & -2.0 & -1.9 & & 6000 & 6 \\
\hline SXCen & B & -0.1 & -0.5 & -2.0 & -2.0 & -1.1 & & 6250 & 6 \\
\hline AC Her & B & -0.4 & -0.9 & -1.7 & -1.6 & -1.4 & -0.7 & 5900 & 4 \\
\hline EP Lyr & B & -0.6 & -0.7 & -2.1 & -2.0 & -1.8 & -0.7 & 6100 & 3 \\
\hline CT Ori & B & -0.5 & -0.6 & -2.6 & -2.5 & -1.9 & -0.6 & 5750 & 1 \\
\hline DY Ori & B & 0.2 & 0.2 & $<-2.7$ & & & +0.2 & 6000 & 3 \\
\hline AR Pup & B & 0.4 & & -2.2 & & & +0.2 & 6000 & 3 \\
\hline DS Aqr & $\mathrm{C}$ & -0.8 & -1.1 & -1.2 & & -1.1 & -1.1 & 6500 & 2 \\
\hline V360 Cyg & $\mathrm{C}$ & -0.9 & -1.4 & & -1.3 & -1.4 & -1.3 & 5300 & 4 \\
\hline BT Lib & $\mathrm{C}$ & -0.8 & -1.1 & -0.7 & & -1.1 & -1.1 & 5800 & 2 \\
\hline V453 Oph & $\mathrm{C}$ & & & -2.2 & -1.9 & -2.2 & -2.2 & 5800 & 4 \\
\hline IRAS 08544 & & 0.1 & 0.1 & -0.9 & -0.9 & -0.3 & 0.0 & 7250 & 6 \\
\hline IRAS 09060 & & -0.7 & -0.5 & -0.8 & -0.7 & -0.7 & -0.7 & 6500 & 6 \\
\hline IRAS 09144 & & 0.0 & & -1.6 & -1.3 & -0.3 & 0.0 & 5750 & 6 \\
\hline IRAS 09538 & A & -0.3 & -0.6 & -0.9 & -0.7 & -0.6 & -0.6 & 5500 & 6 \\
\hline IRAS 15469 & & 0.5 & 0.2 & -1.6 & -1.6 & 0.0 & 0.2 & 7500 & 6 \\
\hline IRAS 16230 & A & -0.4 & -0.4 & -1.3 & -1.4 & -0.7 & -0.4 & 6250 & 6 \\
\hline IRAS 17038 & B & & -1.2 & -2.0 & -1.4 & -1.5 & / & 4750 & 6 \\
\hline IRAS 17233 & B & 0.1 & -0.2 & -1.6 & -1.6 & -1.0 & -0.2 & 6250 & 6 \\
\hline IRAS 17243 & A & 0.0 & 0.2 & -1.1 & -0.6 & 0.0 & 0.2 & 5750 & 6 \\
\hline IRAS 19125 & & 0.5 & 0.1 & $<-1.4$ & $<-2.1$ & -0.3 & 0.1 & 7750 & 6 \\
\hline IRAS 19157 & & 0.2 & & -0.4 & -0.2 & +0.1 & +0.1 & 7750 & 6 \\
\hline IRAS 20056 & & 0.1 & -0.1 & -0.7 & -1.0 & -0.3 & -0.2 & 5850 & 7 \\
\hline HR 4049 & & -0.4 & $\begin{array}{l}-1.3 \\
\end{array}$ & & & -4.8 & -0.4 & 7600 & 8,9 \\
\hline HD 52961 & & -1.0 & -1.4 & & & -4.8 & l & 6000 & 10,11 \\
\hline HD 44179 & & -0.3 & -0.6 & & & -3.3 & -0.6 & 7500 & 8,12 \\
\hline $\mathrm{BD}+39^{\circ} 4926$ & & -0.1 & & -3.0 & -3.3 & -2.9 & -0.1 & 7500 & 13 \\
\hline HD 46703 & & -0.4 & -1.4 & & & -1.6 & -0.4 & 6000 & 14,15 \\
\hline HD 213985 & & 0.4 & & -1.3 & -1.5 & -0.9 & I & 8200 & 8 \\
\hline ST Pup & & -0.2 & -0.1 & -2.2 & -2.2 & -1.5 & -0.1 & 5500 & 16 \\
\hline$\lambda$ Boo & & & & & -2.0 & -2.0 & I & 8650 & 17 \\
\hline
\end{tabular}

(1): Gonzalez et al. (1997a); (2): Giridhar et al. (2000); (3): Gonzalez et al. (1997b); (4): Giridhar et al. (1998); (5): Giridhar et al. (1994); (6): this work; (7) Rao et al. (2002); (8): Van Winckel (1995); (9): Takeda et al. (2002); (10): Waelkens et al. (1991b); (11): Van Winckel et al. (1992); (12): Waelkens et al. (1996); (13): (Kodaira 1973); (14): Luck \& Bond (1984); (15): Bond \& Luck (1987); (16): Gonzalez \& Wallerstein (1996); (17): Venn \& Lambert (1990).

$[\alpha / \mathrm{Fe}]=0.1$ (with $\alpha=\mathrm{Si}, \mathrm{S}, \mathrm{Ca}, \mathrm{Ti}$ ). The C-abundance of $[\mathrm{C} / \mathrm{Fe}]=-0.25$ is rather low. This could be caused by $\mathrm{CNO}-$ cycling on the first giant branch, leading to an $\mathrm{N}$-enhancement. Unfortunately, no good N-lines are present. Neither are good $\mathrm{O}$-lines available. For three s-process elements $\mathrm{Y}, \mathrm{Zr}$ and $\mathrm{La}$ we determined abundances with a mean of +0.12 relative to iron. Since we found no carbon or s-process enhancement, there is no chemical evidence for third dredge-up in IRAS 09060-2807. 


\section{IRAS 09144-4933}

This star is faint and highly reddened which results in a spectrum with a low $S / N$ of 30 . As a consequence the number of used lines is low and we did not use lines in the domain until $4900 \AA$. Unfortunately, no Zn-abundance could be derived. Looking at Fig. 6, we note that up to a certain $T_{\text {cond }}$ no correlation between the abundances and $T_{\text {cond }}$ is present. However, La, Nd, Ti, Y, Sc, all having $T_{\text {cond }}>1500 \mathrm{~K}$, are clearly underabundant by at least one order of magnitude. $\mathrm{Ca}$ complicates the picture, since it has a $T_{\text {cond }}$ of $1624 \mathrm{~K}$ and is not underabundant.

\section{IRAS 09538-7622}

The abundances of this object are based on a CORALIE spectrum, of which the $S / N$ (40) is considerably less than the other spectra. Because also the wavelength domain covered by CORALIE is smaller than that by FEROS, the derived abundances rely on fewer lines and are not as accurate as those of the other stars. A higher $S / N$ spectrum over a larger spectral range is needed to confirm the abundances obtained in this work. Maas et al. (in preparation) identify IRAS 09538-7622 as a genuine RV Tauri star. Its RV Tauri spectroscopic class is A (Lloyd Evans, 1999 and in preparation). Its abundances are not affected by the depletion process and scale roughly with iron. Only $\mathrm{Nd}$ and $\mathrm{Ce}$ seem to be underabundant, but it is not clear with which strength. Since $[\mathrm{Zn} / \mathrm{Fe}]=-0.1$, the low metallicity -0.6 is initial and points to an old nature. This is also indicated by the high measured radial velocity of $97 \mathrm{~km} \mathrm{~s}^{-1}$ of this CORALIE spectrum and the high galactic latitude of $-17.24 \mathrm{deg}$.

\section{IRAS 15469-5311}

The temperature for IRAS 15469-5311 was determined on the basis of Fe II-lines. We believe that the model parameters are not as precise as for the other stars (note the difference in abundance for Fe I and Fe II) so the absolute abundances (certainly the super solar abundances of $\mathrm{S}, \mathrm{Mn}, \mathrm{Si}, \mathrm{Na}, \mathrm{Cu}$ ) should be handled with caution, the relative abundances are considered to be more precise.

For this star we have two spectra, one taken on 23/03/2000, the other on $27 / 06 / 2001$. For both spectra we used the same model parameters, which resulted in similar abundances. We show the abundances of the spectrum taken in 2000 in Fig. 6, which has the highest signal-to-noise.

The high $[\mathrm{S} / \alpha]$ of 0.9 indicates that a depletion process has changed a part of the chemical composition. However, we note that there is a large spread among the underabundances: $[\mathrm{Y} / \mathrm{Fe}]=-0.5$ and $[\mathrm{Ca} / \mathrm{Fe}]=-0.4$ versus $[\mathrm{Ti} / \mathrm{Fe}]=-1.6$ and $[\mathrm{Sc} / \mathrm{Fe}]=-1.6$. Also Ba has a low abundance $([\mathrm{Ba} / \mathrm{Fe}]=-0.6)$, despite its "lower" $T_{\text {cond }}(=1162 \mathrm{~K})$.

\section{IRAS 16230-3410}

$[\mathrm{Zn} / \mathrm{Fe}]=+0.3$ suggest that the iron abundance $([\mathrm{Fe} / \mathrm{H}]=$ -0.7 ) is slightly affected. Abundances of elements up to a
$T_{\text {cond }}$ of about $1500 \mathrm{~K}$ are at most mildly changed by the depletion process. Above that temperature, there is a large spread in underabundances going from -0.1 for $[\mathrm{Ca}, \mathrm{Zr}, \mathrm{Sm} / \mathrm{Fe}]$ to -0.8 for $[\mathrm{Ti} / \mathrm{Fe}]$ and the upperlimit of -1.0 for $[\mathrm{Ce} / \mathrm{Fe}]$. IRAS 16230-3410 is identified as a genuine RV Tauri star (Lloyd Evans, 1999 and in preparation). It belongs to the spectroscopic group A. The depletion process has, at most, effected a moderate change in the chemical composition of the RV Tauri stars of this class (Giridhar et al. 2000). In this way the chemical composition of IRAS 16230-3410 is in agreement with its membership of group A.

\section{IRAS 17038-4815}

IRAS 17038-4815 was noticed by Lloyd Evans (1999 and in preparation) to be an extreme example of an RV Tauri star of spectroscopic group $B$ with by far the strongest $C_{2}$ bands of any B-group star. A spectral classification based on the low-resolution data would indicate a $\mathrm{CH}$ star or possibly a regular $\mathrm{R}$ star. Many $\mathrm{CH}$ stars have even shallower $\mathrm{C}_{2}$ bands. Our analysis based on high-resolution spectra indicate, however, that the object is strongly depleted (Table 6, Fig. 6). Since the s-process elements are not enhanced relative to the species with similar condensation temperature, we have no evidence for 3rd dredge-up enrichment. Unfortunately, good $\mathrm{O}$ lines are not available but despite the fact that the object has a very strong $[\mathrm{C} / \mathrm{Fe}]$ ratio of +1.8 , it is not at all clear if this object is a genuine post-carbon star. This star illustrates the finding that strong depletion can mimic intrinsic carbon enhancement by increasing the $\mathrm{C} / \mathrm{O}$ ratio.

\section{IRAS 17233-4330}

$[\mathrm{Zn} / \mathrm{Fe}]=+0.7$ and $[\mathrm{S} / \alpha]=+1.5$ leaves no doubt about the depleted photosphere of IRAS 17233-4330, which is a genuine RV Tauri star of group B (Lloyd Evans, 1999 and in preparation).

\section{IRAS 17243-4348 (LR Sco)}

For this star, which is considered as a genuine RV Tauri star by Lloyd Evans (1999), we have two spectra at our disposal. The first is taken on 24/03/2000, the second on 28/06/2001, corresponding to different phases in the pulsation cycle. For the first model we find a $T_{\text {eff }}$ of $6250 \mathrm{~K}$ and a $\log g$ of 0.5 for the second a $T_{\text {eff }}$ of $5250 \mathrm{~K}$ and a $\log g$ of 0.0 . To compare the abundances derived from the different spectra, we first intended to use only the same lines in both analyses. However, we dropped this restriction, since due to the large differences in model parameters, the lines in common are too few to derive accurate abundances.

The differences in absolute and relative abundances between both spectra are shown in Table A.1. We note that the differences in relative abundances are smaller than the absolute differences (except for C, S and Y). The agreement between the different relative abundances is for most elements good $(<0.2)$. For C, Sc, Zn, Y and Eu the differences are larger. However the Sc, Zn, Y and Eu abundances are only determined on the basis 
Table A.1. Comparison between the abundances of IRAS 17243-4348 derived from the spectrum taken on 24/03/2000 and that taken on $28 / 06 / 2001 . \Delta[\mathrm{el} / \mathrm{H}]=[\mathrm{el} / \mathrm{H}]_{2001}-[\mathrm{el} / \mathrm{H}]_{2000}, \Delta[\mathrm{el} / \mathrm{Fe}]=[\mathrm{el} / \mathrm{Fe}]_{2001}-$ $[\mathrm{el} / \mathrm{Fe}]_{2000}$. The last column shows the difference of the 2001 relative abundances with those derived by Giridhar et al. (1992): $\Delta[\mathrm{el} / \mathrm{Fe}]_{\mathrm{Gir}}=$ $[\mathrm{el} / \mathrm{Fe}]_{\text {Giridhar }}-[\mathrm{el} / \mathrm{Fe}]_{2001}$.

\begin{tabular}{l|rrr}
\hline \hline ion & $\Delta[\mathrm{el} / \mathrm{H}]$ & $\Delta[\mathrm{el} / \mathrm{Fe}]$ & $\Delta[\mathrm{el} / \mathrm{Fe}]_{\text {Gir }}$ \\
\hline C I & 0.28 & 0.33 & 0.08 \\
Na I & -0.26 & -0.12 & 0.03 \\
Si I & -0.14 & 0.00 & 0.09 \\
S I & 0.04 & 0.09 & 0.24 \\
Ca I & -0.11 & 0.03 & -0.17 \\
Sc II & -0.35 & 0.30 & 0.71 \\
Ti I & & & 0.76 \\
Ti II & -0.08 & 0.03 & 0.58 \\
Cr I & & & 0.08 \\
Cr II & -0.10 & 0.05 & 0.16 \\
Mn I & -0.16 & 0.02 & 0.34 \\
Fe I & -0.14 & & -0.11 \\
Fe II & -0.05 & & -0.19 \\
Ni I & -0.13 & 0.01 & 0.00 \\
Zn I & -0.29 & -0.21 & 0.37 \\
Y II & 0.17 & 0.22 & 1.67 \\
Eu II & -0.26 & -0.21 & \\
\hline
\end{tabular}

of one line. For $\mathrm{C}$ we prefer the abundance of the hot model, since this abundance is based on many more lines.

The ratios $[\mathrm{Zn} / \mathrm{Fe}], 0.24$ and 0.09 , and $[\mathrm{S} / \alpha], 0.21$ and 0.36 , for the 2000 spectrum and 2001 spectrum respectively, are also in good agreement. The underabundances of the s-process elements are also confirmed by the 2000 spectrum. However, the lines, used for the 2001 spectrum, are blended and can not be used for accurate abundance determination.

Figure 6 shows the abundance versus the condensation temperature for the 2001 analysis. The diagram for the 2000 analysis shows the same trends and would lead to the same conclusions. Up to a condensation temperature of about $1500 \mathrm{~K}$, the abundances are approximately solar. The elements with a higher condensation temperature, like the s-process elements, (Ca), Ti and Sc are clearly underabundant. There is, however, a large spread in underabundances. IRAS 17243-4348 belongs to group A (Lloyd Evans, 1999 and in preparation), which is in agreement with its chemical composition.

Giridhar et al. (1992) also made an abundance analysis of IRAS $17243-4348$. They used a $T_{\text {eff }}$ of $5500 \mathrm{~K}$, a $\log g$ of 0.5 and a $\xi_{t}$ of $5.5 \mathrm{~km} \mathrm{~s}^{-1}$. Lines with an $E W$ up to $450 \mathrm{~m} \AA$ are used in their analysis. We compared their relative abundances with ours derived from the spectrum taken in 2001 (see Table A.1), because the used temperatures differ only by $250 \mathrm{~K}$. For $\mathrm{C}, \mathrm{Na}, \mathrm{Si}, \mathrm{Ca}, \mathrm{Cr}, \mathrm{Fe}$ and $\mathrm{Ni}$ the agreement is very good. Worse is the agreement for Sc, Ti, Mn, Zn and Y. For the first two the uncertainty on their abundances is very large $(0.82$ for Sc, 0.29 for Ti I and 0.38 for Ti II). Their Y-abundance is based on one line.

\section{IRAS $19125+0343$}

$[\mathrm{Zn} / \mathrm{Fe}]=+0.4$ and $[\mathrm{S} / \alpha]=+0.8$ point to a depleted photosphere.

\section{IRAS 19157-0247}

Many low excitation lines appear in emission in this object. These were not taken into account for the abundance analysis. As a consequence, abundances are determined for few elements in IRAS 19157-0247. Fe is approximately solar, no clear trend is seen in the abundance-condensation temperature diagram and the underabundances are not large $([\mathrm{Ti} / \mathrm{Fe}]=-0.3,[\mathrm{Sc} / \mathrm{Fe}]=-0.5)$. In this way the evidence for a depleted photosphere for IRAS 19157-0247 is lacking.

\section{IRAS 20056+1834 (QY Sge)}

The abundances of this object are taken from Rao et al. (2002). They do not limit their lines towards a low $E W$. For Ba, for example, they use the $5853 \AA$ line with an $E W$ of $288 \mathrm{~m} \AA$. We can see in Fig. 6 that they obtain very similar trends to those we observe for IRAS 09144-4933, IRAS 15469-5311, IRAS 16230-3410, IRAS 17243-4348 and IRAS 19125+0343. They note that "there is a clear tendency for the elements with a high $T_{\text {cond }}$ to be underabundant relative to those of low $T_{\text {cond". }}$ " Furthermore, they remark that "the abundances anomalies are reminiscent of those observed in stars of group B, but that, in contrast, iron and other elements are only slightly, if at all, depleted". 